\title{
Indirect dark matter detection for flattened dwarf galaxies
}

\author{
Jason L. Sanders, ${ }^{1, *}$ N. Wyn Evans, ${ }^{1, \dagger}$ Alex Geringer-Sameth, ${ }^{2, \$}$ and Walter Dehnen ${ }^{3, \$}$ \\ ${ }^{1}$ Institute of Astronomy, Madingley Rd, Cambridge CB3 OHA, United Kingdom \\ ${ }^{2}$ McWilliams Center for Cosmology, Department of Physics, Carnegie Mellon University, \\ Pittsburgh, Pennsylvania 15213, USA \\ ${ }^{3}$ Department of Physics \& Astronomy, University of Leicester, \\ University Road, Leicester LE1 7RH, United Kigndom \\ (Received 2 April 2016; published 15 September 2016)
}

\begin{abstract}
Gamma-ray experiments seeking to detect evidence of dark matter annihilation in dwarf spheroidal galaxies require knowledge of the distribution of dark matter within these systems. We analyze the effects of flattening on the annihilation (J) and decay (D) factors of dwarf spheroidal galaxies with both analytic and numerical methods. Flattening has two consequences: first, there is a geometric effect as the squeezing (or stretching) of the dark matter distribution enhances (or diminishes) the J-factor; second, the line of sight velocity dispersion of stars must hold up the flattened baryonic component in the flattened dark matter halo. We provide analytic formulas and a simple numerical approach to estimate the correction to the $\mathrm{J}$ - and D-factors required over simple spherical modeling. The formulas are validated with a series of equilibrium models of flattened stellar distributions embedded in flattened dark-matter distributions. We compute corrections to the J- and D-factors for the Milky Way dwarf spheroidal galaxies under the assumption that they are all prolate or all oblate and find that the hierarchy of J-factors for the dwarf spheroidals is slightly altered (typical correction factors for an ellipticity of 0.4 are 0.75 for the oblate case and 1.6 for the prolate case). We demonstrate that spherical estimates of the D-factors are very insensitive to the flattening and introduce uncertainties significantly less than the uncertainties in the D-factors from the other observables

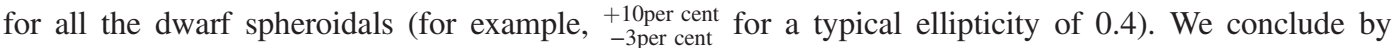
investigating the spread in correction factors produced by triaxial figures and provide uncertainties in the J-factors for the dwarf spheroidals using different physically motivated assumptions for their intrinsic shape and axis alignments. We find that the uncertainty in the J-factors due to triaxiality increases with the observed ellipticity and, in general, introduces uncertainties of a factor of 2 in the J-factors. We discuss our results in light of the reported gamma-ray signal from the highly flattened ultrafaint Reticulum II. Tables of the J- and D-factors for the Milky Way dwarf spheroidal galaxies are provided (assuming an oblate or prolate structure) along with a table of the uncertainty on these factors arising from the unknown triaxiality.
\end{abstract}

DOI: 10.1103/PhysRevD.94.063521

\section{INTRODUCTION}

In recent years, gamma-ray observations of Milky Way dwarf spheroidal galaxies (dSphs) have led to great strides in sensitivity to dark matter annihilation. Here the goal is to probe particles which interact with the Standard Model with the well-motivated weak-scale annihilation cross section $\langle\sigma v\rangle \simeq 3 \times 10^{-26} \mathrm{~cm}^{3} \mathrm{~s}^{-1}$. Particles having this cross section will exist today with an abundance equal to that observed for dark matter $\Omega_{\mathrm{DM}}$, making this so-called relic cross section a natural target for experimental searches for annihilation. Combined analyses of dSphs using data from the Fermi Large Area Telescope (LAT) first ruled out the relic cross section for dark matter particle masses of a few tens of $\mathrm{GeV}[1,2]$ and follow-up analyses incorporating more dSphs and increased observation time continue to

\footnotetext{
jils@ast.cam.ac.uk

nwe@ast.cam.ac.uk

*alexgs@cmu.edu

$\S_{\text {wd11@leicester.ac.uk }}$
}

improve sensitivity (e.g. Refs. [3-5]). For higher dark matter masses $(M \gtrsim \mathrm{TeV})$, the three major Cherenkov telescope collaborations continue to invest significant time on pointed observations of Milky Way dSphs. The resulting upper limits are 2 to 3 orders of magnitude from the relic cross section [6-10], but the situation bodes well for the future CTA project (e.g. Ref. [11]).

An exciting development in this field is the recent and ongoing discovery of large numbers of new Milky Way satellites made possible by wide-area photometric surveys (e.g. Refs. [12-15]). Since 2015 the number of known Milky Way satellites has approximately doubled thanks to Southern hemisphere data from the Dark Energy Survey and Pan-STARRS. These new dSphs have the potential to significantly build on current efforts to uncover evidence of dark matter annihilation (e.g. Refs. [16-20]).

Intriguingly, the first of these new dwarf spheroidal galaxies discovered, Reticulum II, shows indications of a gamma-ray signal exceeding background in the Fermi-LAT data [17]. Two methods of modeling the gamma-ray 
background yield false-alarm probabilities of $p=0.0001$ and 0.01 for detecting such a signal. Subsequent analysis [19] confirmed the results of Ref. [17] and argued that the Reticulum II signal was consistent with the gamma-ray excess reported from the Galactic center and claimed as dark matter. With a reprocessing of the raw Fermi data [18], the Fermi-LAT Collaboration found an increased probability for a background fluctuation explaining the Reticulum II signal $(p=0.05)$ and concluded the signal is insignificant. Making sense of the results of Refs. [17] and [18] is complicated by the fact that the two data sets are only partially independent, sharing approximately half the detector events. A separate analysis is needed to compute joint probabilities of background fluctuation in the partially correlated data sets.

In this work we follow a different path towards assessing dark matter interpretations of gamma-ray signals. Rather than analyzing the gamma-ray data, we consider the determination of the dark matter content of the Milky Way's dSphs, a necessary ingredient for performing optimized combined searches using dSphs. A critical test of any alleged dark matter signal from dSphs is that the amplitude of the gamma-ray signal must scale amongst the dSphs according to their J-factors (see, e.g., Refs. [21,22]). The J-factor is the square of the dark matter density integrated along the line of sight and over the solid angle of the observation,

$$
J=\iint \rho_{\mathrm{DM}}^{2}(\ell, \Omega) d \ell d \Omega
$$

While annihilating dark matter models are theoretically better motivated, there are models in which dark matter decays [23]. In these models, the relevant astrophysical factor is the D-factor, which is the dark matter density integrated along the line of sight and over the solid angle of the observation.

Robust determinations of the relative J-factors are of prime importance. For instance, the Fermi-LAT Collaboration [18], under the assumption that each of eight considered dSphs was equally likely to produce a signal, further diluted the significance of the Reticulum II gamma-ray excess to $p=1-(1-0.05)^{8}=0.33$, concluding that it is insignificant. However, there are reasons to doubt the usefulness of this argument as Reticulum II is closer and very highly flattened, both of which can enhance the amplitude of an annihilation signal compared to other dSphs. Therefore, we require accurate relative estimates of the $\mathrm{J}$ - and $\mathrm{D}$-factors, but unfortunately the data on the most tempting $\mathrm{dSph}$ candidates are often of limited quality. Motivated by this Ref. [24] (hereafter Paper I) provided simple formulas for the J- and D-factors for a spherical Navarro-Frenk-White (NFW) profile and infinite spherical cusps. The formulas relied on the empirical law that the mass within the half-light radius is well constrained as $[25,26]$

$$
M_{\mathrm{h}}=M\left(R_{\mathrm{h}}\right) \approx \frac{5}{2 G}\left\langle\sigma_{\text {los }}^{2}\right\rangle R_{\mathrm{h}},
$$

where $R_{\mathrm{h}}$ is the (projected) half-light radius of the stars and $\left\langle\sigma_{\text {los }}^{2}\right\rangle$ is the luminosity weighted squared line-of-sight velocity dispersion.

However, an entirely characteristic feature of dSphs is in the name-spheroidal! They are flattened (with a typical ellipticity between 0.3 and 0.5 ), and some of the ultrafaints are very highly flattened with ellipticities exceeding 0.5 , such as Hercules [27], Ursa Major I [28], Ursa Major II [29], and indeed Reticulum II [12]. Therefore, the underlying physical model of a spherical dark halo containing a round distribution of stars may fail to capture important aspects of the physics. Here we extend the scope of spherical analyses, to account for the effects of flattening in both the stellar and dark matter profiles. Bonnivard et al. [30] provided a systematic investigation of J-factors of flattened figures. Here, two mildly triaxial numerical models of dSphs (created for The Gaia Challenge) were viewed along each of the short, medium and long axes. This investigation revealed that the projection effects can have a significant impact on the velocity dispersion, and concluded that the J-factors constructed by Jeans analyses can vary from the true values by $\sim 2.5$. Recently, Ref. [31] computed J-factor estimates for the dSphs using axisymmetric Jeans modeling. These authors attributed the differences between their measured J-factors and those from spherical analyses primarily to other modeling assumptions.

It is natural to expect that the dissipationless dark matter distribution is rounder - or at least no more flattened - than the dissipative baryonic component. So, large classical dSphs which appear roundish on the sky (such as Leo I and II) may have almost spherical dark matter halos. However, the dark halos of the ultrafaints are expected to be more highly flattened than those of the classical dSphs, as it is known that baryonic feedback effects drive the dark matter distribution towards sphericity $[32,33]$. The ultrafaints have such a puny baryonic content that pure dissipationless simulations [34,35], which find strongly triaxial and nearly prolate dark halos, may be a much better guide to the true shape. For instance, recent simulations have found that the baryonic distribution is just $\sim 10$ percent flatter than the dark-matter distribution for dark-matter halos of $10^{10} M_{\odot}$ [36]. Throughout this paper, we work under the assumption that the dark matter distribution is flattened in the same way as the stellar distribution.

The effects of flattening can be understood qualitatively for a few simple configurations. The simplest is the face-on case when the dark-matter and stellar distributions are flattened along the line of sight. Observationally, the isophotes still appear circular and the measured half-light radius remains the same, but we have increased (decreased) the density of dark matter in the oblate (prolate) case. 
Naturally, this effect-which we refer to as the geometric factor-gives rise to a larger (smaller) J-factor than a spherical analysis would infer. But, we must also consider the effect of flattening on the line-of-sight velocity dispersion, which we call the kinematic factor. For the oblate case, the stellar distribution is more compressed, so the line-of-sight dispersion is now smaller than the spherically averaged dispersion. Less contained mass is inferred and so the spherical J-factor underestimates the total $\mathrm{J}$-factor. Therefore, for face-on viewing of an oblate figure, both the geometric and kinematic effects cause the J-factor inferred from a spherical analysis to be an underestimate of the true value. For the prolate case, the velocity dispersion is larger than the spherically averaged dispersion and so more mass is inferred and the spherical $\mathrm{J}$-factor is an overestimate.

When the dSph is viewed edge on such that it appears flattened in the sky, the combined result of the kinematic and geometric effects is less clear. For oblate figures, the density is increased over the spherical case, while the halflight radius remains the same. These geometric effects cause the J-factor assuming sphericity to be an underestimate. However, the kinematic factor works the other way, as the measured velocity dispersion is greater than the spherical average. We will see that the combination of these two competing effects leads to a small decrease in the true J-factor over that inferred from a spherical analysis. For the prolate case, we have the converse situation with the geometric factor leading to an overestimate while the kinematic factor leads to an underestimate. However, now the stretching of the stellar profile in the sky causes the half-light radius to increase. We will see that the net result is an increase in the true J-factor over the spherical J-factor.

This qualitative explanation is tested in Sec. II where we construct equilibrium models of the Reticulum II galaxy via the made-to-measure method. We explore a range of different flattenings and provide simple fits for the correction factors. In Sec. III, we use these fits to derive J-factors for the known dwarf spheroidals under the assumption that they are either prolate or oblate. In Sec. IV, we build intuition for our numerical results by considering two families of axisymmetric equilibria for which analytic progress is possible and present a more rapid general approach for estimating the correction factors using the virial theorem. Section $\mathrm{V}$ extends these findings to the triaxial case and demonstrates how the correction factors vary for a triaxial figure as a function of the viewing angle. In Sec. VI, we discuss the constraints and evidence on the intrinsic shapes and alignments of the Milky Way dSphs and give estimates of the uncertainties in the J-factors of the dSphs due to unknown triaxiality. In Sec. VII we summarize our findings and discuss possible implications for the claimed signal from Reticulum II in light of our work.

\section{MADE-TO-MEASURE FLATTENED EQUILIBRIA}

We begin our analysis of the J-factors of flattened dSphs with numerical models constructed by the made-tomeasure (M2M) methods [37] as implemented by Dehnen [38]. The models have two components: dark and stellar. Each component has a target density of the form

$\rho(m) \propto p^{-1} q^{-1}\left(\frac{m}{r_{\mathrm{s}}}\right)^{-\gamma}\left(1+\left(\frac{m}{r_{\mathrm{s}}}\right)^{\alpha}\right)^{(\gamma-\beta) / \alpha} \operatorname{sech} \frac{m}{r_{\mathrm{t}}}$,

where $m^{2}=x^{2}+(y / p)^{2}+(z / q)^{2}$. This is the familiar double power law with scale radius $r_{\mathrm{s}}$, with an exponential taper at the tidal radius $r_{\mathrm{t}}$. For $r \ll r_{\mathrm{s}}$, the density falls like $r^{-\gamma}$, while for $r \gg r_{\mathrm{s}}$, it falls like $r^{-\beta}$. The case $\alpha=1$, $\beta=3, \gamma=1$ is the NFW dark halo. Plummer models are often used to describe the light profiles of dSphs (see e.g., Refs. [39,40]). They correspond to the parameters $\alpha=2$, $\beta=5, \gamma=1$ and $r_{\mathrm{t}}=\infty$.

We begin by constructing two flattened spheroidal $(p=1)$ models of the Reticulum II dSph. For both models, the dark halo is a NFW model $(\alpha=1, \beta=3, \gamma=1$, $\left.r_{s}=1, r_{\mathrm{t}}=10\right)$. The stars follow a Plummer profile $\left(\alpha=2, \beta=5, \gamma=0, r_{s}=0.5, r_{t}=9\right)$. The chosen ratio of the dark matter scale radius to the stellar scale radius lies within the measured range for the Local Group dSphs $(\sim 1.25$ to $~ 30)$ [41]. The two models differ in their shape. The first model is oblate in both the stars and the dark matter with an axis ratio of $q=0.4$ (chosen to match the observed axis ratio of Reticulum II of 0.39 [12]). The second model is prolate with an axis ratio of $q=2.5$. When viewed along the $x$ axis both models appear flattened with axis ratio 0.4 . In addition, we construct a third spherical model as a reference. This has the same parameters, but without the flattening in either the dark matter or the stars.

The dark NFW halos source the potential (computed using a bi-orthonormal basis expansion [38]) in which the weights of the Plummer models are adjusted until the target densities are reached. No other constraints on the distribution functions are used. We use a $10^{7}$ particle realization of the flattened NFW distribution to compute the potential. The constraints on the Plummer model are generated with 100 realizations of $10^{6}$ particles and $10^{6}$ particles are used in the M2M simulation. To check convergence, the models were run turning off the weight adjustment in the M2M code. Both flattened models exhibit a slow drift in the density constraint suggesting they are not perfect equilibrium models. However, this is almost certainly true for the actual dSphs which reside in the tidal field of the Milky Way.

Reticulum II has a half-light major axis length of $5.63 \mathrm{arcmin}$, is at a distance of $\sim 30 \mathrm{kpc}$ [12] and has a line-of-sight velocity dispersion of $3.22 \mathrm{~km} \mathrm{~s}^{-1}$ [42]. To match the final models to the observed constraints on Reticulum II, we compute the projected half-light major 
axis length (fitted with a Plummer model) and the projected line-of-sight velocity dispersion. We then compute the scale factors $\mathcal{R}$ and $\mathcal{V}$ that scale the radial distributions and the velocity distributions to the observations. The corresponding total mass of the dark matter profile (set to unity in the simulation) is then scaled by a factor $\mathcal{M}=\mathcal{R} \mathcal{V}^{2}$. For the spherical model we match the half-light major axis length to an "ellipticity corrected" radius given by the geometric mean of the half-light major and minor axis lengths. This is related to the observed half-light major axis length $R_{\mathrm{h}}$ as $R_{\mathrm{h}} \sqrt{1-\epsilon}$ where $\epsilon$ is the ellipticity.

In Fig. 1, we show the final projected distributions of the two flattened models. Note that for the prolate case, the models do not completely reproduce the target density profile as there is a clear $\mathrm{X}$ shape in the $(x, y)$ plane. Additionally, we show the surface density of the two models (using a mass-to-light ratio of 500, [42]) and the line-of-sight velocity distributions. The prolate velocity distribution is slightly more peaked than the oblate case but such a small difference would not be detectable observationally.

To explore the effects of adjusting the stellar and darkmatter profiles, we also build two further models, one with a central cusp in the stellar profile $(\gamma=1)$ and one with a cored dark-matter profile with parameters $\alpha=1, \beta=4$ and $\gamma=0$.

\section{A. J- and D-factors}

For our five models of Reticulum II, we proceed to calculate the $\mathrm{J}$ - and $\mathrm{D}$-factors. The $\mathrm{J}$-factor for a distant source is given by ${ }^{1}$

$$
\mathrm{J}(\theta)=\frac{1}{D^{2}} \int_{-\infty}^{+\infty} \mathrm{d} z \int_{0}^{D \theta} \mathrm{d} R R \int_{0}^{2 \pi} \mathrm{d} \phi \rho_{\mathrm{DM}}^{2},
$$

where $D$ is the distance to the source ( $30 \mathrm{kpc}$ for Reticulum II) and $\theta$ is the beam angle. Similarly, the D-factor is given by

$$
\mathrm{D}(\theta)=\frac{1}{D^{2}} \int_{-\infty}^{+\infty} \mathrm{d} z \int_{0}^{D \theta} \mathrm{d} R R \int_{0}^{2 \pi} \mathrm{d} \phi \rho_{\mathrm{DM}}
$$

In Table I we report the $\mathrm{J}$ - and D-factors at $\theta=0.5^{\circ}$ (the typical observational resolution). We also show the $\mathrm{J}$ - and D-factors for the spherical model computed from the formulas of Paper I. We see that these formulas underestimate the $\mathrm{J}$-factor by a factor of 1.2 and the $\mathrm{D}$-factor by a factor of 1.05. We also record the correction factor between the prolate/oblate models and the spherical models using the notation

\footnotetext{
${ }^{1}$ When computing these integrals numerically, we have found it useful to perform the coordinate transformation $\tan \chi=z / r_{s}$ where $r_{s}$ is the scale radius of the density profile.
}

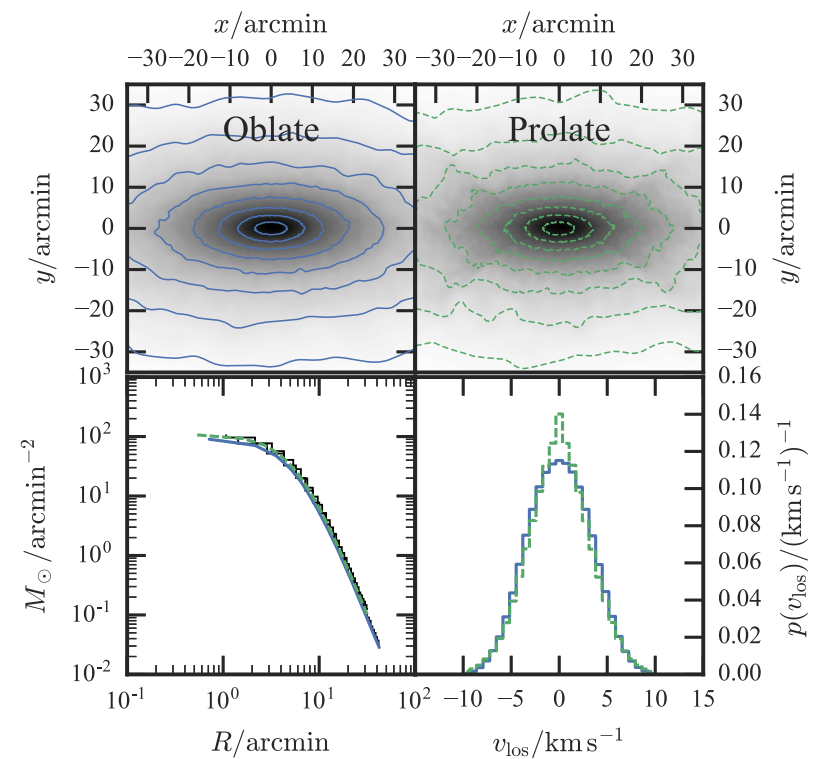

FIG. 1. Reticulum II M2M equilibria of a flattened Plummer distribution of stars in a flattened NFW dark halo. The top left panel shows the logarithm of the projected mass distribution of an oblate model viewed edge on with axis ratio 0.4 . The contours are logarithmically spaced. The top right panel shows the logarithm of the projected mass distribution of a prolate model viewed edge on with axis ratio 0.4 . Note the X-shape in the prolate case. The bottom left panel shows the surface density profiles in elliptical bins with Plummer profile fits (oblate in blue, prolate in dashed green). The bottom right panel shows the line-of-sight velocity distributions (oblate in blue, prolate in dashed green).

$$
\begin{aligned}
\mathcal{F}_{\mathrm{J}} & =\log _{10}\left(\mathrm{~J} / \mathrm{J}_{\mathrm{sph}}\right), \\
\mathcal{F}_{\mathrm{D}} & =\log _{10}\left(\mathrm{D} / \mathrm{D}_{\text {sph }}\right) .
\end{aligned}
$$

The oblate model with NFW dark matter and Plummer light has a J-factor that is diminished by a factor of 1.4 over the spherical model and a D-factor that is diminished by a factor of 1.3. On the other hand, the prolate model has an enhancement in the J-factor by a factor of 3.4 and a small decrease in the D-factor of 10 percent. The near-prolate model with a cuspy stellar profile produces a very similar $\mathrm{J}$-factor to the Plummer prolate model, but here the D-factor is enhanced over the spherical model by 20 percent. Finally, in a similar fashion to the prolate NFW profile, the prolate cored dark matter profile also produces an enhancement in the $\mathrm{J}$-factor of a factor of 3 and a small diminution in the D-factor of order 10 percent.

\section{B. A range of flattenings}

We have established that a prolate model of Reticulum II viewed edge on produces a significant enhancement in the J-factor over its spherical counterpart, while an oblate model has a slight diminution. However, the observed dSphs span a whole range of ellipticities, so we now go on to explore models with a variety of flattenings. We 
TABLE I. J- and D-factors for a beam angle of $0.5^{\circ}$ for a series of Reticulum II models. The J-factors are in units of $\mathrm{GeV}^{2} \mathrm{~cm}^{-5}$ and the $\mathrm{D}$-factors are in units of $\mathrm{GeV} \mathrm{cm}{ }^{-2}$. Each model was normalized such that the line-of-sight velocity dispersion and half-light major axis length matched that of Reticulum II. For the spherical model, an "ellipticity corrected" half-light radius of $R_{\mathrm{h}} \sqrt{1-\epsilon}$ (where $\epsilon$ is the ellipticity) was used to scale the models. Note the correction factors are with respect to the spherical NFW, spherical Plummer model in the first row and not with respect to the corresponding spherical model.

\begin{tabular}{|c|c|c|c|c|c|c|}
\hline Model & Paper I J & Paper I D & $\log _{10}\left(\mathrm{~J}\left(0.5^{\circ}\right)\right)$ & $\log _{10}\left(\mathrm{D}\left(0.5^{\circ}\right)\right)$ & $\mathcal{F}_{\mathrm{J}}$ & $\mathcal{F}_{\mathrm{D}}$ \\
\hline $\begin{array}{l}\text { Spherical NFW } \\
\text { Spherical Plummer }\end{array}$ & 18.56 & 17.56 & 18.64 & 17.58 & 0.00 & 0.00 \\
\hline $\begin{array}{l}\text { Oblate NFW, } p=1, q=0.4 \\
\text { Oblate Plummer, } p=1, q=0.4\end{array}$ & $\ldots$ & $\ldots$ & 18.45 & 17.62 & -0.19 & 0.05 \\
\hline $\begin{array}{l}\text { Prolate NFW, } p=1, q=2.5 \\
\text { Prolate Plummer, } p=1, q=2.5\end{array}$ & $\ldots$ & $\ldots$ & 19.05 & 17.67 & 0.40 & 0.09 \\
\hline $\begin{array}{l}\text { Near-prolate NFW, } p=0.5, q=0.4 \\
\text { Near-prolate cuspy Plummer } \alpha_{\star}=2, \\
\quad \beta_{\star}=5, \gamma_{\star}=1, p=0.4, q=0.38\end{array}$ & $\ldots$ & $\ldots$ & 19.01 & 17.78 & 0.37 & 0.20 \\
\hline $\begin{array}{l}\text { Prolate cored DM } \alpha_{\mathrm{DM}}=1, \beta_{\mathrm{DM}}=4 \\
\quad \gamma_{\mathrm{DM}}=0, p=1, q=2.5 \\
\text { Prolate Plummer, } p=1, q=2.5\end{array}$ & $\ldots$ & $\ldots$ & 18.90 & 17.66 & 0.26 & 0.08 \\
\hline
\end{tabular}

construct three oblate M2M models with the same parameters as the spherical reference model in Table I but with flattenings $q=0.5,0.6,0.7$, and similarly three prolate M2M models with flattenings $q=1.423,1.667,2$. Again the M2M models are normalized to match the line-of-sight velocity dispersion and half-light major-axis length of Reticulum II.

The J- and D-factors for our series of models are plotted in Fig. 2. All models are viewed such that they appear maximally flattened (along the short axis for the prolate cases and along the long axis for the oblate cases). We also show the J- and D-factors computed using the simple formulas [Eqs. (15) and (19)] from Paper I. We see that this formula disagrees with the spherical case by $\sim 0.2$ due to the use of the empirical relation for the half-light mass. As shown in Paper I, for most dSphs this is less than the uncertainty in the J-factor due to uncertainties in the lineof-sight velocity dispersion and half-light radius.

The prolate models produce a sequence of more enhanced J-factors at all angles as we increase the flattening $q$. The oblate models produce a similar sequence of decreasing $\mathrm{J}$ as we decrease the flattening $q$. These trends are reproduced in the D-factor. Note the asymmetry with $q$ in both $\mathrm{J}$ and $\mathrm{D}$ : the equivalent flattening for a prolate model produces a larger difference from the spherical model than the corresponding oblate model.

With this sequence of models, we also investigate how the J-factor for an apparently round dSph changes as the $\mathrm{dSph}$ is flattened along the line of sight. In Fig. 3, we show the range of $\mathrm{J}$ - and D-factors for the set of flattened models viewed face on such that the isophotes appear round and all models have the same half-light radius. We see that the range of possible J-factors with flattening along the line of sight varies by a factor of 10 . The oblate models all have a similar decrease in the J-factor. The D-factor is unaffected by flattening along the line of sight.
For the series of flattened M2M models, we compute the correction factors $\mathcal{F}_{\mathrm{J}}$ and $\mathcal{F}_{\mathrm{D}}$ by comparing each model with the spherical model with the same line-of-sight velocity dispersion and the "ellipticity corrected" half-light

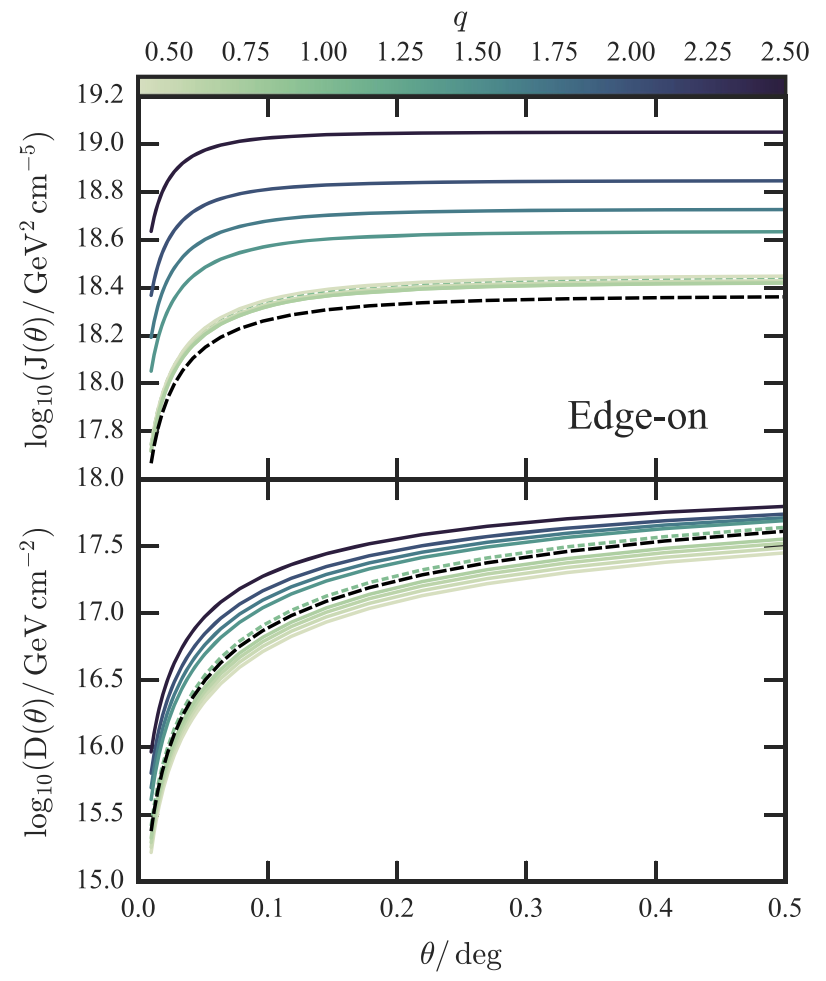

FIG. 2. J-and D-factors as a function of beam angle for a range of flattened models viewed edge on with identical line-of-sight velocity dispersions and half-light major-axis lengths. The models are colored by the flattening in the density of both the stars and dark matter, $q$. The spherical model is shown with the short-dashed line, while the analytic formula for the NFW model [Eqs. (15) and (19) from Paper I] is shown with the long-dashed line. 


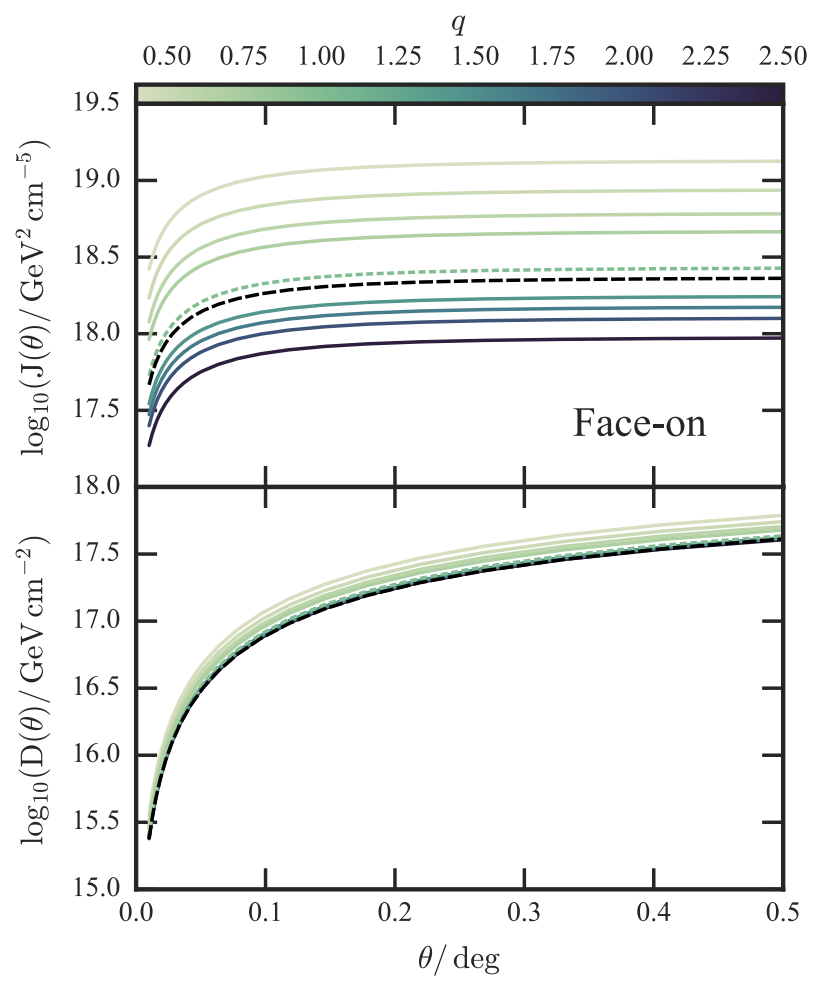

FIG. 3. J- and D-factors as a function of beam angle for a range of flattened models viewed face on with identical line-of-sight velocity dispersions and half-light major-axis lengths. The models are colored by the flattening in the density of both the stars and dark matter, $q$. The spherical model is shown with the short-dashed line and the model using the formulas from Paper I is shown with the long-dashed line.

radius. The trends of $\mathcal{F}_{\mathrm{J}}$ and $\mathcal{F}_{\mathrm{D}}$ with respect to $q$ are very smooth so we opt to fit the corrections from the models with a simple functional form

$$
\mathcal{F}_{\text {fit }}=\eta \log _{10}(q)
$$

where we fit $q<1$ and $q>1$ separately. The values of $\eta$ chosen are given in Table II. Although our fit is an

TABLE II. Slopes $\eta$ of the base-10 logarithms of the correction factors with respect to $\log _{10} q$ fitted to the made-to-measure models of Sec. II. The prolate and oblate cases are treated separately. The multiplicative factor by which a J- or D-factor from a spherical analysis must be corrected is given by $q^{\eta}$. Note the spherical models to which we compare use an "ellipticity corrected" half-light radius of $R_{\mathrm{h}} \sqrt{1-\epsilon}$ where $\epsilon=1-q$ is the ellipticity in the oblate case and $\epsilon=1-1 / q$ in the prolate case.

\begin{tabular}{llcc}
\hline \hline & View & $\eta$ Oblate $(q<1)$ & $\eta$ Prolate $(q>1)$ \\
\hline $\mathcal{F}_{\mathrm{J}}$ & Edge on & 0.534 & 0.899 \\
& Face on & -1.647 & -1.181 \\
$\mathcal{F}_{\mathrm{D}}$ & Edge on & 0.056 & 0.177 \\
& Face on & -0.335 & -0.089 \\
\hline \hline
\end{tabular}

extrapolation for $q<0.4$ and $q>2.5$, we will see that it agrees well with the more involved models of Sec. IV. In reality, the correction factors are functions of the beam angle. We have found that the correction factors are very insensitive to the beam angle so this formula is appropriate for all dSphs irrespective of their size compared to the resolution of the instrument.

\section{J- AND D-FACTORS FOR THE MILKY WAY DSPHS}

We now apply the corrections to the J-and D-factors of the observed dSphs. They are listed in Table III along with their measured ellipticities $\epsilon=1-b / a$ where $b / a$ is the observed axis ratio. We take the majority of the ellipticities and $\pm 1 \sigma$ error bars from the review of Ref. [43]. The ellipticities of the new dSphs discovered in the Dark Energy Survey are taken from Ref. [12], the ellipticity of Pisces II is taken from Ref. [44] and that of Hydra II from Ref. [45]. For both Leo T and Horologium I, only upper bounds on the ellipticity are available.

For each $\mathrm{dSph}$, we compute the correction factor assuming the $\mathrm{dSph}$ is either oblate or prolate and observed edge on. We draw samples from the error distributions of the ellipticities and compute the median and $\pm 1 \sigma$ values of the correction factors for both the J- and D-factors using Eq. (7). The baseline spherical model to which we are comparing uses an effective half-light radius of $R_{\mathrm{h}} \sqrt{1-\epsilon}$. We combine these estimates with the spherical estimates computed in Paper I (adding the errors in quadrature). The results of this procedure are reported in Tables III and IV.

We show this data in Fig. 4. We plot the distribution of $\mathrm{J}$ - and D-factors for the dSphs assuming they are spherical, oblate or prolate. Ursa Major I has the largest ellipticity and hence the largest prolate correction factor (a factor of $\sim 4$ ). Reticulum II, Ursa Major II and Hercules all have ellipticities $\sim 0.6$ and so the prolate correction factors are approximately $\sim 2.2-2.7$. For ellipticities less than $\sim 0.4$, the correction factors are less than the errors on the spherical $\mathrm{J}$-factors. For every dSph the correction to the D-factors is smaller than the errors in the spherical D-factor. Hence, we conclude that flattening has a negligible effect on the D-factor estimates.

If the entire population of $\mathrm{dSphs}$ is prolate then only Tucana II and Willman 1 have potentially higher J-factors than Reticulum II, with both Ursa Major II and Segue 1 having a very similar J-factor to Reticulum II. We remark that Tucana II is consistent with having circular isophotes [12], while the assumption of dynamical equilibrium for Willman 1 is dubious [46]. Similarly, Ursa Major II appears to be in the process of severe tidal disruption [29]. Finally, the J-factor of Segue 1 has been shown to be extremely sensitive to the presence of foreground contaminants (e.g., Refs. $[47,48])$. These final three dSphs have been marked in red in Fig. 4 to indicate their dubious J-factors. Therefore, it 
TABLE III. Annihilation correction factors for dwarf spheroidals due to their observed ellipticity $\epsilon$ (note Leo T and Horologium I only have upper bounds on the ellipticity). We report the spherical J-factor for a beam angle of $0.5^{\circ}$ in units of $\mathrm{GeV}^{2} \mathrm{~cm}^{-5}$ along with the corrections $\mathcal{F}_{\mathrm{J}}$ assuming the galaxy is observed exactly edge on and is either oblate or prolate. We report the resultant $\mathrm{J}$-factors for these cases as $\mathrm{J}_{\mathrm{obl}}$ and $\mathrm{J}_{\text {pro }}$ in units of $\mathrm{GeV}^{2} \mathrm{~cm}^{-5}$. The dSphs are ordered by their ellipticity.

\begin{tabular}{|c|c|c|c|c|c|c|}
\hline Name & $\epsilon$ & $\log _{10}\left(\mathrm{~J}_{\mathrm{sph}}\left(0.5^{\circ}\right)\right)$ & Oblate $\mathcal{F}_{\mathrm{J}}$ & Prolate $\mathcal{F}_{\mathrm{J}}$ & $\log _{10}\left(\mathrm{~J}_{\mathrm{obl}}\left(0.5^{\circ}\right)\right)$ & $\log _{10}\left(J_{\text {pro }}\left(0.5^{\circ}\right)\right)$ \\
\hline Hydra II & $0.01_{-0.01}^{+0.20}$ & $16.56_{-1.85}^{+0.87}$ & $-0.002_{-0.052}^{+0.002}$ & $0.004_{-0.003}^{+0.087}$ & $16.56_{-1.85}^{+0.87}$ & $16.56_{-1.85}^{+0.87}$ \\
\hline Leo $\mathrm{T}$ & $<0.10$ & $17.32_{-0.37}^{+0.38}$ & $-0.012_{-0.009}^{+0.008}$ & $0.020_{-0.014}^{+0.015}$ & $17.31_{-0.37}^{+0.38}$ & $17.34_{-0.37}^{+0.38}$ \\
\hline Leo II & $0.13_{-0.05}^{+0.05}$ & $17.44_{-0.25}^{+0.25}$ & $-0.032_{-0.017}^{+0.011}$ & $0.054_{-0.018}^{+0.029}$ & $17.41_{-0.25}^{+0.25}$ & $17.49_{-0.25}^{+0.25}$ \\
\hline Segue 2 & $0.15_{-0.10}^{+0.10}$ & $17.11_{-1.76}^{+0.85}$ & $-0.037_{-0.042}^{+0.019}$ & $0.063_{-0.032}^{+0.070}$ & $17.07_{-1.76}^{+0.85}$ & $17.17_{-1.76}^{+0.85}$ \\
\hline Leo I & $0.21_{-0.03}^{+0.03}$ & $17.80_{-0.28}^{+0.28}$ & $-0.055_{-0.010}^{+0.008}$ & $0.092_{-0.014}^{+0.017}$ & $17.75_{-0.28}^{+0.28}$ & $17.89_{-0.28}^{+0.28}$ \\
\hline Horologium I & $<0.28$ & $18.64_{-0.39}^{+0.95}$ & $-0.034_{-0.028}^{+0.024}$ & $0.058_{-0.040}^{+0.047}$ & $18.61_{-0.39}^{+0.95}$ & $18.70_{-0.39}^{+0.95}$ \\
\hline Fornax & $0.30_{-0.01}^{+0.01}$ & $18.15_{-0.16}^{+0.16}$ & $-0.083_{-0.003}^{+0.003}$ & $0.139_{-0.005}^{+0.006}$ & $18.07_{-0.16}^{+0.16}$ & $18.29_{-0.16}^{+0.16}$ \\
\hline Draco & $0.31_{-0.02}^{+0.02}$ & $18.86_{-0.24}^{+0.24}$ & $-0.086_{-0.007}^{+0.006}$ & $0.145_{-0.011}^{+0.012}$ & $18.77_{-0.24}^{+0.24}$ & $19.00_{-0.24}^{+0.24}$ \\
\hline Sculptor & $0.32_{-0.03}^{+0.03}$ & $18.65_{-0.29}^{+0.29}$ & $-0.090_{-0.011}^{+0.010}$ & $0.151_{-0.016}^{+0.019}$ & $18.56_{-0.29}^{+0.29}$ & $18.80_{-0.29}^{+0.29}$ \\
\hline Carina & $0.33_{-0.05}^{+0.05}$ & $17.99_{-0.34}^{+0.34}$ & $-0.093_{-0.020}^{+0.016}$ & $0.156_{-0.026}^{+0.034}$ & $17.90_{-0.34}^{+0.34}$ & $18.15_{-0.34}^{+0.34}$ \\
\hline Sextans & $0.35_{-0.05}^{+0.05}$ & $17.87_{-0.29}^{+0.29}$ & $-0.100_{-0.020}^{+0.016}$ & $0.169_{-0.028}^{+0.034}$ & $17.77_{-0.29}^{+0.29}$ & $18.04_{-0.29}^{+0.29}$ \\
\hline Coma Berenices & $0.38_{-0.14}^{+0.14}$ & $18.67_{-0.32}^{+0.33}$ & $-0.108_{-0.071}^{+0.039}$ & $0.182_{-0.066}^{+0.120}$ & $18.56_{-0.33}^{+0.33}$ & $18.85_{-0.33}^{+0.35}$ \\
\hline Boötes I & $0.39_{-0.06}^{+0.06}$ & $16.65_{-0.38}^{+0.64}$ & $-0.114_{-0.026}^{+0.020}$ & $0.192_{-0.033}^{+0.043}$ & $16.54_{-0.38}^{+0.64}$ & $16.84_{-0.38}^{+0.64}$ \\
\hline Canes Venatici I & $0.39_{-0.03}^{+0.03}$ & $17.27_{-0.11}^{+0.11}$ & $-0.115_{-0.012}^{+0.011}$ & $0.194_{-0.018}^{+0.020}$ & $17.15_{-0.11}^{+0.11}$ & $17.46_{-0.11}^{+0.11}$ \\
\hline Tucana II & $0.39_{-0.20}^{+0.10}$ & $19.05_{-0.58}^{+0.87}$ & $-0.114_{-0.049}^{+0.053}$ & $0.193_{-0.090}^{+0.082}$ & $18.94_{-0.58}^{+0.87}$ & $19.24_{-0.59}^{+0.87}$ \\
\hline Pisces II & $0.40_{-0.10}^{+0.10}$ & $17.90_{-0.80}^{+1.14}$ & $-0.119_{-0.048}^{+0.032}$ & $0.200_{-0.053}^{+0.081}$ & $17.78_{-0.80}^{+1.14}$ & $18.10_{-0.80}^{+1.14}$ \\
\hline Grus I & $0.41_{-0.28}^{+0.20}$ & $17.96_{-1.93}^{+0.90}$ & $-0.117_{-0.117}^{+0.064}$ & $0.198_{-0.107}^{+0.196}$ & $17.84_{-1.93}^{+0.90}$ & $18.16_{-1.93}^{+0.92}$ \\
\hline Willman 1 & $0.47_{-0.08}^{+0.08}$ & $19.29_{-0.62}^{+0.91}$ & $-0.147_{-0.040}^{+0.030}$ & $0.247_{-0.051}^{+0.067}$ & $19.14_{-0.62}^{+0.91}$ & $19.54_{-0.62}^{+0.91}$ \\
\hline Segue 1 & $0.48_{-0.13}^{+0.13}$ & $19.41_{-0.40}^{+0.39}$ & $-0.151_{-0.076}^{+0.045}$ & $0.255_{-0.076}^{+0.128}$ & $19.26_{-0.41}^{+0.39}$ & $19.66_{-0.41}^{+0.41}$ \\
\hline Leo IV & $0.49_{-0.11}^{+0.11}$ & $16.64_{-0.90}^{+0.90}$ & $-0.156_{-0.064}^{+0.041}$ & $0.262_{-0.068}^{+0.108}$ & $16.48_{-0.90}^{+0.90}$ & $16.90_{-0.90}^{+0.91}$ \\
\hline Leo V & $0.50_{-0.15}^{+0.15}$ & $16.94_{-0.72}^{+1.05}$ & $-0.159_{-0.096}^{+0.053}$ & $0.268_{-0.089}^{+0.161}$ & $16.78_{-0.73}^{+1.05}$ & $17.21_{-0.73}^{+1.06}$ \\
\hline Canes Venatici II & $0.52_{-0.11}^{+0.11}$ & $17.65_{-0.40}^{+0.40}$ & $-0.171_{-0.065}^{+0.042}$ & $0.288_{-0.071}^{+0.109}$ & $17.48_{-0.41}^{+0.40}$ & $17.94_{-0.41}^{+0.41}$ \\
\hline Ursa Minor & $0.56_{-0.05}^{+0.05}$ & $19.15_{-0.24}^{+0.25}$ & $-0.191_{-0.029}^{+0.024}$ & $0.321_{-0.040}^{+0.049}$ & $18.96_{-0.24}^{+0.25}$ & $19.47_{-0.24}^{+0.25}$ \\
\hline Reticulum II & $0.59_{-0.03}^{+0.02}$ & $18.71_{-0.32}^{+0.84}$ & $-0.207_{-0.012}^{+0.016}$ & $0.348_{-0.027}^{+0.020}$ & $18.50_{-0.32}^{+0.84}$ & $19.06_{-0.32}^{+0.84}$ \\
\hline Ursa Major II & $0.63_{-0.05}^{+0.05}$ & $19.38_{-0.39}^{+0.39}$ & $-0.231_{-0.035}^{+0.028}$ & $0.389_{-0.047}^{+0.060}$ & $19.15_{-0.39}^{+0.39}$ & $19.77_{-0.39}^{+0.39}$ \\
\hline Hercules & $0.68_{-0.08}^{+0.08}$ & $16.83_{-0.45}^{+0.45}$ & $-0.266_{-0.069}^{+0.050}$ & $0.447_{-0.084}^{+0.116}$ & $16.56_{-0.46}^{+0.45}$ & $17.28_{-0.46}^{+0.46}$ \\
\hline Ursa Major I & $0.80_{-0.04}^{+0.04}$ & $18.48_{-0.25}^{+0.25}$ & $-0.373_{-0.053}^{+0.041}$ & $0.629_{-0.070}^{+0.089}$ & $18.11_{-0.26}^{+0.25}$ & $19.11_{-0.26}^{+0.27}$ \\
\hline
\end{tabular}

is possible that the Reticulum II gamma-ray signal may be due to annihilation if the dwarf has a prolate shape. We can robustly conclude from Fig. 4 that if Reticulum II has a prolate shape then an observed annihilation signal from only Reticulum II is not in tension with the lack of signals from all the other dSphs irrespective of their shapes. If, however, Reticulum II is oblate and has an observed annihilation signal we begin to have some tension if there is a lack of signal from the other dSphs. The majority of this tension arises from those problematic dSphs already mentioned. However, if both Ursa Minor and Tucana II have prolate shapes it becomes unlikely that they both have smaller $\mathrm{J}$ factors than an oblate Reticulum II.

\section{SEMIANALYTIC MODELS}

Numerical M2M models provide a robust method for determining the corrections required when modeling flattened systems as spherical. However, they are computationally expensive to construct so they cannot be employed in a Markov chain Monte Carlo analysis that requires many models. We have provided a simple fitting formula for our model setup, but there will be some variation in the correction factors depending on, for instance, the light profile, the density profile of the dark matter, and the ratio of the scale lengths of the light to the dark matter.

We now proceed to understand and reproduce the results of the $\mathrm{M} 2 \mathrm{M}$ models using simpler methods. In 
TABLE IV. As in Table III, but for the decay correction factors. The D-factors are quoted in units of $\mathrm{GeV} \mathrm{cm}^{-2}$.

\begin{tabular}{|c|c|c|c|c|c|c|}
\hline Name & $\epsilon$ & $\log _{10}\left(\mathrm{D}_{\mathrm{sph}}\left(0.5^{\circ}\right)\right)$ & Oblate $\mathcal{F}_{\mathrm{D}}$ & Prolate $\mathcal{F}_{\mathrm{D}}$ & $\log _{10}\left(\mathrm{D}_{\mathrm{obl}}\left(0.5^{\circ}\right)\right)$ & $\log _{10}\left(D_{\text {pro }}\left(0.5^{\circ}\right)\right)$ \\
\hline Hydra II & $0.01_{-0.01}^{+0.20}$ & $16.89_{-0.92}^{+0.44}$ & $-0.000_{-0.005}^{+0.000}$ & $0.001_{-0.001}^{+0.017}$ & $16.89_{-0.92}^{+0.44}$ & $16.89_{-0.92}^{+0.44}$ \\
\hline Leo $\mathrm{T}$ & $<0.10$ & $17.35_{-0.37}^{+0.37}$ & $-0.001_{-0.001}^{+0.001}$ & $0.004_{-0.003}^{+0.003}$ & $17.35_{-0.37}^{+0.37}$ & $17.35_{-0.37}^{+0.37}$ \\
\hline Leo II & $0.13_{-0.05}^{+0.05}$ & $17.62_{-0.25}^{+0.25}$ & $-0.003_{-0.002}^{+0.001}$ & $0.011_{-0.004}^{+0.006}$ & $17.62_{-0.25}^{+0.25}$ & $17.63_{-0.25}^{+0.25}$ \\
\hline Segue 2 & $0.15_{-0.10}^{+0.10}$ & $17.08_{-1.75}^{+0.86}$ & $-0.004_{-0.004}^{+0.002}$ & $0.012_{-0.006}^{+0.014}$ & $17.08_{-1.75}^{+0.86}$ & $17.09_{-1.75}^{+0.86}$ \\
\hline Leo I & $0.21_{-0.03}^{+0.03}$ & $17.89_{-0.28}^{+0.28}$ & $-0.006_{-0.001}^{+0.001}$ & $0.018_{-0.003}^{+0.003}$ & $17.88_{-0.28}^{+0.28}$ & $17.91_{-0.28}^{+0.28}$ \\
\hline Horologium I & $<0.28$ & $17.78_{-0.20}^{+0.47}$ & $-0.004_{-0.003}^{+0.002}$ & $0.011_{-0.008}^{+0.009}$ & $17.78_{-0.20}^{+0.47}$ & $17.79_{-0.20}^{+0.47}$ \\
\hline Fornax & $0.30_{-0.01}^{+0.01}$ & $18.26_{-0.17}^{+0.17}$ & $-0.009_{-0.000}^{+0.000}$ & $0.027_{-0.001}^{+0.001}$ & $18.25_{-0.17}^{+0.17}$ & $18.29_{-0.17}^{+0.17}$ \\
\hline Draco & $0.31_{-0.02}^{+0.02}$ & $18.39_{-0.25}^{+0.25}$ & $-0.009_{-0.001}^{+0.001}$ & $0.029_{-0.002}^{+0.002}$ & $18.38_{-0.25}^{+0.25}$ & $18.42_{-0.25}^{+0.25}$ \\
\hline Sculptor & $0.32_{-0.03}^{+0.03}$ & $18.33_{-0.29}^{+0.29}$ & $-0.009_{-0.001}^{+0.001}$ & $0.030_{-0.003}^{+0.004}$ & $18.32_{-0.29}^{+0.29}$ & $18.36_{-0.29}^{+0.29}$ \\
\hline Carina & $0.33_{-0.05}^{+0.05}$ & $17.98_{-0.34}^{+0.34}$ & $-0.010_{-0.002}^{+0.002}$ & $0.031_{-0.005}^{+0.007}$ & $17.97_{-0.34}^{+0.34}$ & $18.01_{-0.34}^{+0.34}$ \\
\hline Sextans & $0.35_{-0.05}^{+0.05}$ & $18.07_{-0.29}^{+0.29}$ & $-0.011_{-0.002}^{+0.002}$ & $0.033_{-0.005}^{+0.007}$ & $18.06_{-0.29}^{+0.29}$ & $18.10_{-0.29}^{+0.29}$ \\
\hline Coma Berenices & $0.38_{-0.14}^{+0.14}$ & $18.06_{-0.32}^{+0.32}$ & $-0.011_{-0.007}^{+0.004}$ & $0.036_{-0.013}^{+0.024}$ & $18.05_{-0.32}^{+0.32}$ & $18.10_{-0.32}^{+0.32}$ \\
\hline Boötes I & $0.39_{-0.06}^{+0.06}$ & $17.28_{-0.38}^{+0.64}$ & $-0.012_{-0.003}^{+0.002}$ & $0.038_{-0.007}^{+0.008}$ & $17.27_{-0.38}^{+0.64}$ & $17.32_{-0.38}^{+0.64}$ \\
\hline Canes Venatici I & $0.39_{-0.03}^{+0.03}$ & $17.78_{-0.11}^{+0.11}$ & $-0.012_{-0.001}^{+0.001}$ & $0.038_{-0.004}^{+0.004}$ & $17.77_{-0.11}^{+0.11}$ & $17.82_{-0.11}^{+0.11}$ \\
\hline Tucana II & $0.39_{-0.20}^{+0.10}$ & $18.45_{-0.58}^{+0.88}$ & $-0.012_{-0.005}^{+0.006}$ & $0.038_{-0.018}^{+0.016}$ & $18.44_{-0.58}^{+0.88}$ & $18.49_{-0.58}^{+0.88}$ \\
\hline Pisces II & $0.40_{-0.10}^{+0.10}$ & $17.41_{-0.40}^{+0.57}$ & $-0.012_{-0.005}^{+0.003}$ & $0.039_{-0.010}^{+0.016}$ & $17.40_{-0.40}^{+0.57}$ & $17.45_{-0.40}^{+0.57}$ \\
\hline Grus I & $0.41_{-0.28}^{+0.20}$ & $17.59_{-0.96}^{+0.46}$ & $-0.012_{-0.012}^{+0.007}$ & $0.039_{-0.021}^{+0.039}$ & $17.58_{-0.96}^{+0.46}$ & $17.63_{-0.96}^{+0.46}$ \\
\hline Willman 1 & $0.47_{-0.08}^{+0.08}$ & $18.03_{-0.62}^{+0.91}$ & $-0.015_{-0.004}^{+0.003}$ & $0.049_{-0.010}^{+0.013}$ & $18.01_{-0.62}^{+0.91}$ & $18.08_{-0.62}^{+0.91}$ \\
\hline Segue 1 & $0.48_{-0.13}^{+0.13}$ & $18.17_{-0.39}^{+0.39}$ & $-0.016_{-0.008}^{+0.005}$ & $0.050_{-0.015}^{+0.025}$ & $18.15_{-0.39}^{+0.39}$ & $18.22_{-0.39}^{+0.39}$ \\
\hline Leo IV & $0.49_{-0.11}^{+0.11}$ & $17.22_{-0.90}^{+0.90}$ & $-0.016_{-0.007}^{+0.004}$ & $0.052_{-0.013}^{+0.021}$ & $17.20_{-0.90}^{+0.90}$ & $17.27_{-0.90}^{+0.90}$ \\
\hline Leo V & $0.50_{-0.15}^{+0.15}$ & $17.23_{-0.70}^{+1.05}$ & $-0.017_{-0.010}^{+0.006}$ & $0.053_{-0.018}^{+0.032}$ & $17.21_{-0.70}^{+1.05}$ & $17.28_{-0.70}^{+1.05}$ \\
\hline Canes Venatici II & $0.52_{-0.11}^{+0.11}$ & $17.37_{-0.40}^{+0.40}$ & $-0.018_{-0.007}^{+0.004}$ & $0.057_{-0.014}^{+0.022}$ & $17.35_{-0.40}^{+0.40}$ & $17.43_{-0.40}^{+0.40}$ \\
\hline Ursa Minor & $0.56_{-0.05}^{+0.05}$ & $18.45_{-0.24}^{+0.24}$ & $-0.020_{-0.003}^{+0.002}$ & $0.063_{-0.008}^{+0.010}$ & $18.43_{-0.24}^{+0.24}$ & $18.51_{-0.24}^{+0.24}$ \\
\hline Reticulum II & $0.59_{-0.03}^{+0.02}$ & $17.93_{-0.32}^{+0.85}$ & $-0.022_{-0.001}^{+0.002}$ & $0.069_{-0.005}^{+0.004}$ & $17.91_{-0.32}^{+0.85}$ & $18.00_{-0.32}^{+0.85}$ \\
\hline Ursa Major II & $0.63_{-0.05}^{+0.05}$ & $18.48_{-0.39}^{+0.39}$ & $-0.024_{-0.004}^{+0.003}$ & $0.077_{-0.009}^{+0.012}$ & $18.46_{-0.39}^{+0.39}$ & $18.56_{-0.39}^{+0.39}$ \\
\hline Hercules & $0.68_{-0.08}^{+0.08}$ & $17.38_{-0.45}^{+0.45}$ & $-0.028_{-0.007}^{+0.005}$ & $0.088_{-0.017}^{+0.023}$ & $17.35_{-0.45}^{+0.45}$ & $17.47_{-0.45}^{+0.45}$ \\
\hline Ursa Major I & $0.80_{-0.04}^{+0.04}$ & $18.15_{-0.25}^{+0.25}$ & $-0.039_{-0.006}^{+0.004}$ & $0.124_{-0.014}^{+0.018}$ & $18.11_{-0.25}^{+0.25}$ & $18.27_{-0.25}^{+0.25}$ \\
\hline
\end{tabular}

subsection A, we describe a general virial method to compute J-factors for flattened halo models. This numerical algorithm can be applied to any dark matter density, but in the two following subsections, we provide analytic shortcuts to evaluate the J-factors for two specific families: the infinite flattened cusps and the flat rotation curve halos. Readers primarily interested in the results, rather than the details of the methods, should skip to subsection $\mathrm{D}$, where we compare our models to the M2M results. Figures 6 and 7 provide summary plots, which show the range of correction factors as a function of the flattening of the stellar density.

\section{A. The virial method}

We can construct approximate equilibrium models much more cheaply than with the full M2M apparatus by using the virial theorem. The two constraints provided by the data are the integrated line-of-sight velocity dispersion $\left\langle\sigma_{\text {los }}^{2}\right\rangle$ and the half-light major-axis length $R_{h}$. We describe a method to match these observations given density models for the light $\left(\rho_{\star}\right)$ and dark matter $\left(\rho_{\mathrm{DM}}\right)$.

(1) For a given viewing angle $(\vartheta, \varphi)$, we find the measured ellipticity and orientation of the observed minor axis [using, for instance, Eqs. (A1), (A2), and (A6) of Ref. [49]] and compute the elliptical half-light radius $R_{h}^{\prime}$. This gives us a length scaling $\mathcal{R}=R_{h} / R_{h}^{\prime}$, and encodes the geometric factor described in the Introduction.

(2) In principle, to solve for the kinematics of the stars in the dark matter potential, we could use the axisymmetric Jeans equations. However, there are degeneracies in the solution space and only a few algorithms exist for a solution [50,51]. As we need 

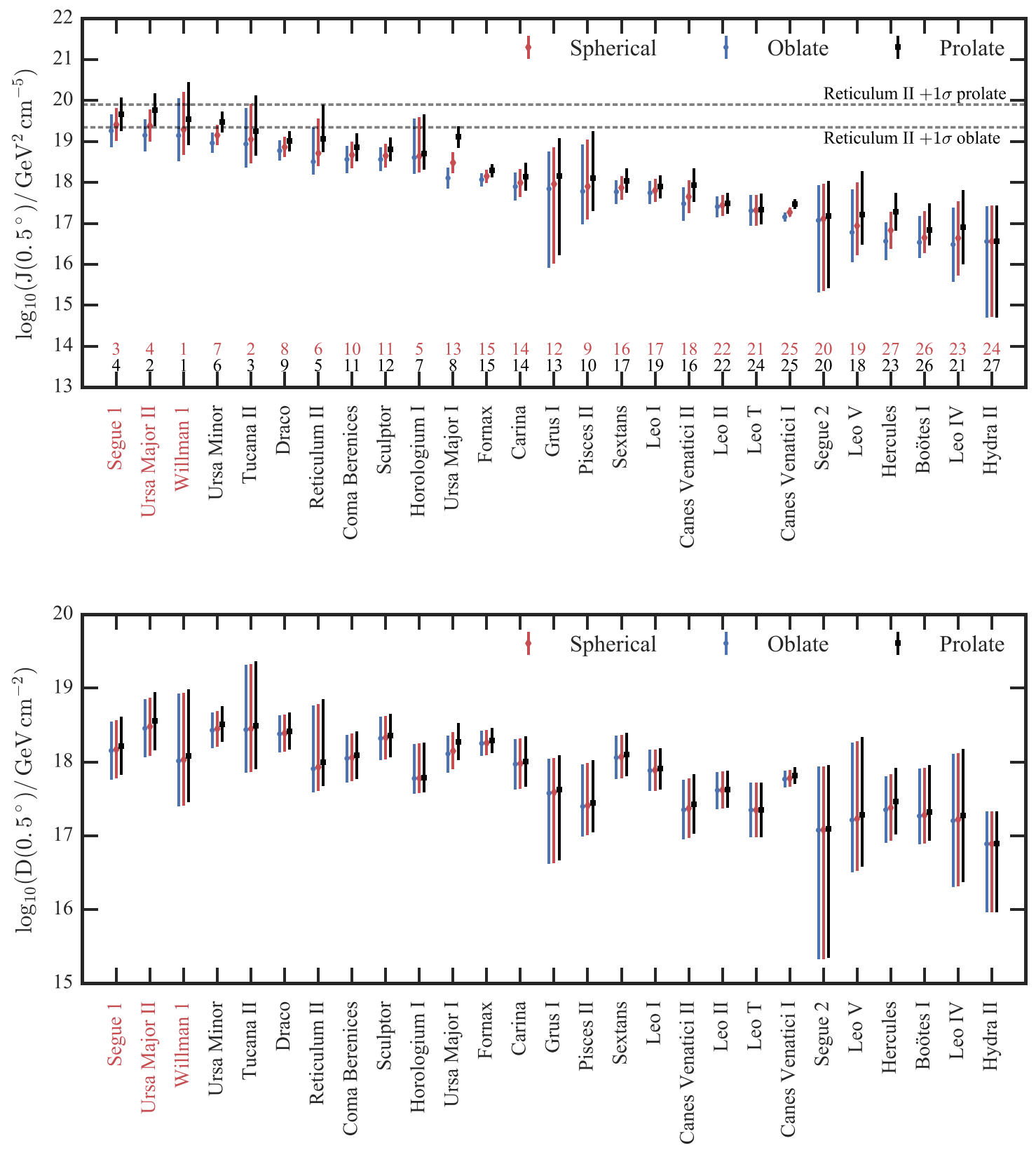

FIG. 4. J- (top) and D-factors (bottom) integrated over a beam angle of $0.5^{\circ}$ for $27 \mathrm{dSphs}$. The diamonds with red error bars are computed assuming a spherical model and are taken from Paper I. The circles with blue error bars show the spherical J-factors adjusted by the oblate correction factors marginalized over the uncertainty in the ellipticity (assuming the galaxy is observed edge on) and the squares with black error bars show the spherical J-factors adjusted by the prolate correction factors marginalized over the uncertainty in the ellipticity (assuming the galaxy is observed edge on). The dSphs are ordered by their median spherical J-factors. The top set of red numbers gives the ordering of the upper limits on the spherical J-factors, and the bottom set of black numbers gives the ordering of the upper limits on the prolate J-factors. The gray dashed lines show the $1 \sigma$ upper limit for the Reticulum II assuming it is prolate or oblate. The three dSphs with red names have unknown additional systematic uncertainties due to the presence of contaminants or the questionable assumption of dynamical equilibrium.

only match an integrated quantity, we use the virial theorem to compute $\left\langle\sigma_{\mathrm{los}}^{2}\right\rangle$ as

$$
\left\langle\sigma_{\text {los }}^{\prime 2}\right\rangle=\frac{W_{\text {los }}}{W}\left\langle\sigma_{\text {tot }}^{2}\right\rangle=\frac{W_{\text {los }}}{M},
$$

where

$$
W_{\mathrm{los}}=\int \mathrm{d}^{3} \boldsymbol{x} \rho_{\star} R_{i j} x_{j} \frac{\partial \Phi_{\mathrm{DM}}}{\partial x_{k}} R_{k i} .
$$

$\Phi_{\mathrm{DM}}$ is the dark matter potential (generically computed using a multipole expansion [52]), $M$ is the total dark matter mass and $R_{i j}$ is the projection 
matrix along the line-of-sight from coordinates aligned with the principal axes of the dSph. We have used the Einstein summation convention. For triaxial symmetry, the cross terms in the integral vanish so we need only project the velocity dispersions along the principal axes. This gives us a velocity scaling $\mathcal{V}=\left\langle\sigma_{\text {los }}^{2}\right\rangle /\left\langle\sigma_{\text {los }}^{\prime 2}\right\rangle$, and encodes the kinematic factor described in the Introduction.

(3) We compute a mass scaling $\mathcal{M}=\mathcal{V}^{2} \mathcal{R}$. The initial model is scaled by $\mathcal{M}$ and $\mathcal{R}$ and the $\mathrm{J}$ - and $D$-factors are computed. These can be compared to the spherical model with the same line-of-sight velocity dispersion and half-light radius.

This algorithm is completely general. For some special choices of stellar and dark matter density, the integration in the virial theorem can be performed analytically. We now give two examples - infinite flattened cusps and flat rotation curve halos-for which the virial integrals can be done. This means that the behavior of the J-factor at fixed observables (line-of-sight velocity dispersion and half-light radii) can be mapped out analytically as a function of flattening or concentration.

\section{B. Flattened cusps}

Let us take the dark matter halo as an axisymmetric cusp stratified on similar concentric spheroids with an axis ratio $q$. If the cusps have the same mass $M_{\mathrm{h}}$ within the spheroidal half-light radius $m_{\mathrm{h}}$, then the mass enclosed is

$$
M(m)=M_{\mathrm{h}}\left(\frac{m}{m_{\mathrm{h}}}\right)^{3-\gamma_{\mathrm{DM}}} \text { for } \quad m \leq r_{t}
$$

and $\quad M=M_{\mathrm{h}}\left(r_{t} / m_{\mathrm{h}}\right)^{3-\gamma_{\mathrm{DM}}} \quad$ otherwise. $\quad m^{2}=x^{2}+y^{2}+$ $z^{2} q^{-2}=R^{2}+z^{2} q^{-2}$ and $r_{t}$ is a hard truncation ellipsoidal radius. The dark matter density is

$$
\rho_{\mathrm{DM}}(m)=\frac{M_{\mathrm{h}}}{4 \pi q m_{\mathrm{h}}^{3-\gamma_{\mathrm{DM}}}} \frac{3-\gamma_{\mathrm{DM}}}{m^{\gamma_{\mathrm{DM}}}} \text { for } \quad m \leq r_{t},
$$

and zero otherwise. Note the factor of $q$ in the denominator which comes from the Jacobian. It means that the oblate models $(q<1)$ in the sequence have an increased density as compared to their spherical progenitor, while the prolate models $(q>1)$ have a decreased density. The spherical member of the family obeys the empirical law (2). As the mass $M_{\mathrm{h}}$ is preserved along the sequence, we can still use Eq. (2) for the flattened cusps provided we correct the observables - the line-of-sight velocity dispersion and the projected half-light radius - to the spherical parent.

For comparison purposes, it is useful to define the $\mathrm{J}$-factor and D-factor of the infinite spherical cusp $\left[r_{t} \rightarrow \infty\right.$, Eqs. (8) and (11) in Paper I] as

$$
\begin{array}{r}
\mathrm{J}_{\mathrm{sph}}=\frac{1}{D^{2} R_{\mathrm{h}}^{3}}\left(\frac{\left\langle\sigma_{\text {los }}^{2}\right\rangle R_{\mathrm{h}}}{G}\right)^{2}\left(\frac{D \theta}{R_{\mathrm{h}}}\right)^{3-2 \gamma_{\mathrm{DM}}} P\left(\gamma_{\mathrm{DM}}\right), \\
\mathrm{D}_{\mathrm{sph}}=\frac{1}{D^{2}} \frac{\left\langle\sigma_{\mathrm{los}}^{2}\right\rangle R_{\mathrm{h}}}{G}\left(\frac{D \theta}{R_{\mathrm{h}}}\right)^{3-\gamma_{\mathrm{DM}}} Q\left(\gamma_{\mathrm{DM}}\right),
\end{array}
$$

where both $P\left(\gamma_{\mathrm{DM}}\right)$ and $Q\left(\gamma_{\mathrm{DM}}\right)$ are constants given in Paper I. In these expressions, the half-light radius $R_{\mathrm{h}}$ is the "ellipticity corrected" half-light radius that includes a factor of $\sqrt{1-\epsilon}$. The J- and D- factors for our axisymmetric models can now be written in the form

$$
\begin{gathered}
\mathrm{J}=\mathrm{J}_{\text {sph }} \mathrm{J}_{\text {geo }} \mathrm{J}_{\text {kin }}, \\
\mathrm{D}=\mathrm{D}_{\text {sph }} \mathrm{D}_{\text {geo }} \mathrm{D}_{\text {kin }} .
\end{gathered}
$$

If an oblate model is viewed along the short axis, or a prolate model is viewed along the long axis, then it appears round. The line of sight coincides with the symmetry or $z$ axis. The geometric corrections are then straightforward to evaluate as

$$
\mathrm{J}_{\text {geo,face }}=\frac{1}{q}, \quad \mathrm{D}_{\text {geo,face }}=1 .
$$

This case is very simple because both the field of view and the surface density contours are circular. Note that both factors are independent of the slope of the density profile $\gamma_{\mathrm{DM}}$.

If an infinite $\left(r_{t} \rightarrow \infty\right)$ oblate or prolate model is viewed edge on, it appears flattened with axis ratio $q$. The line of sight then coincides with, say, the $y$ direction. Observationally, the effective radius of a flattened model is always measured along the projected major axis. For an oblate model, the measured effective radius is $R_{\mathrm{h}}$ whereas for the prolate model, the effective radius of its spherical progenitor is actually $R_{\mathrm{h}} / q$. Additionally, comparison with the "ellipticity corrected" spherical model gives rise to an additional factor of $\sqrt{1-\epsilon}$ in the effective radius which equals $\sqrt{q}$ for the oblate case and $\sqrt{1 / q}$ for the prolate case.

The geometric corrections (i.e. the ratio of the J- and D-factors to those for a spherical model with the same $M_{\mathrm{h}}$ ) are now

$\mathrm{J}_{\text {geo,edge }}=\frac{q^{2-\gamma_{\mathrm{DM}}}}{2 \pi q^{2}} \int_{0}^{2 \pi} d \theta\left(\cos ^{2} \theta+q^{-2} \sin ^{2} \theta\right)^{1 / 2-\gamma_{\mathrm{DM}}}$,

and

$\mathrm{D}_{\text {geo }, \text { edge }}=\frac{q^{1-\gamma_{\mathrm{DM}} / 2}}{2 \pi q} \int_{0}^{2 \pi} d \theta\left(\cos ^{2} \theta+q^{-2} \sin ^{2} \theta\right)^{1 / 2-\gamma_{\mathrm{DM}} / 2}$. 
The factors can only be reduced to a single quadrature due to the mismatch between the circular beam aperture and the elliptical isophotes. For oblate (prolate) models, the geometric correction leads to an increase (decrease) in the $\mathrm{J}$-factor as compared to a spherical model with the same $M_{\mathrm{h}}$ if $\gamma_{\mathrm{DM}} \leq 2$. If the dark matter halo is truncated at a finite ellipsoidal radius $r_{t}<D \theta$, the beam encloses all the dark matter and the edge-on geometric factors reduce to

$$
\mathrm{J}_{\text {geo,edge }}=q^{1-\gamma_{\mathrm{DM}}}, \quad \mathrm{D}_{\text {geo,edge }}=q^{1-\gamma_{\mathrm{DM}} / 2} .
$$

These equations are preferable as for $\gamma_{\mathrm{DM}}<3$ they correspond to finite mass models and for $\gamma_{\mathrm{DM}}<3 / 2$ they produce finite $\mathrm{J}$-factors. We have found that they give much better representations of the correction factors for more general models.

We have computed the ratio of the J-and D-factors to those of the spherical model with the same $M_{\mathrm{h}}$. As $M_{\mathrm{h}}$ is estimated from the line-of-sight velocity dispersion, we must now compute the ratio of the true $M_{\mathrm{h}}$ to that computed using only the line-of-sight velocity dispersion. This ratio is the kinematic correction, which we compute using the tensor virial theorem $[53,54]$. The effect of flattening on the kinematics of the stars is given by

$$
\frac{T_{R R}}{T_{z z}}=\frac{W_{R R}}{W_{z z}}=\frac{\int \mathrm{d}^{3} \boldsymbol{x} \rho_{\star}\left(\sigma_{R R}^{2}+\sigma_{\phi \phi}^{2}\right)}{\int \mathrm{d}^{3} \boldsymbol{x} \rho_{\star} \sigma_{z z}^{2}}
$$

where $T$ and $W$ are the kinetic energy and potential energy tensors [52,54,55]. The stellar density in dSphs is well approximated by a Plummer or King profile. Such laws do not lead to tractable integrals in the virial theorem (19). Instead, we approximate the stellar density as a power-law stratified on similar concentric spheroids with $m_{\star}^{2}=R^{2}+$ $z^{2} q_{\star}^{-2}$ and so $q_{\star}$ is the stellar flattening. This means we can take advantage of Eqs. (19)-(24) in Ref. [54], which give the virial ratios for stellar populations whose density is a pure scale-free power law declining like distance ${ }^{-\gamma_{\star}}$. Note that as all the considered models have infinite mass we must work with the ratios of the velocity dispersions.

If we assume the equipotentials are spheroidally stratified, the correction is a function of $Q_{\star}=q_{\phi}^{2} / q_{\star}^{2}$, where $q_{\phi}$ is the flattening of the dark halo equipotentials, which is related to the flattening $q$ in the dark halo density via

$$
q_{\phi}=\frac{1}{2}\left(1+\left(1+8 q^{2}\right)^{1 / 2}\right)^{1 / 2} .
$$

This formula is given in Refs. [56,57]. As is well known, the equipotentials are always rounder than the density contours, so that $q_{\phi} \approx 1$ even if the dark halo is quite flattened. Then for $\gamma_{\star}=3$, we have from Ref. [54]

$$
\frac{\left\langle\sigma_{x x}^{2}\right\rangle}{\left\langle\sigma_{z z}^{2}\right\rangle}= \begin{cases}\frac{Q_{\star} \mathcal{Q}-\sqrt{Q_{\star}} \operatorname{Arcsinh} \mathcal{Q}}{2\left[\sqrt{Q_{\star}} \operatorname{Arcsinh} \mathcal{Q}-\mathcal{Q}\right]}, & Q_{\star}>1 \\ \frac{Q_{\star} \mathcal{Q}-\sqrt{Q_{\star}} \operatorname{Arcsin} \mathcal{Q}}{2\left[\sqrt{Q_{\star}} \operatorname{Arcsin} \mathcal{Q}-\mathcal{Q}\right]}, & Q_{\star}<1,\end{cases}
$$

where

$$
\mathcal{Q}\left(Q_{\star}\right)= \begin{cases}\sqrt{Q_{\star}-1} & Q_{\star}>1 \\ \sqrt{1-Q_{\star}} & Q_{\star}<1\end{cases}
$$

Our formulas are appropriate if $r_{t} \rightarrow \infty$ or the stellar profile is truncated at a smaller radius than the dark-matter profile. This virial ratio is unity when $Q_{\star}=1$. This follows because if the stellar density is constant on the equipotentials, then the velocity dispersion is isotropic. It is greater than unity when $Q_{\star}>1$ (that is, when the model is oblate), as the globally averaged velocity dispersion component along the long or $x$ axis must be larger than that along the short or $z$ axis. It is less than unity when $Q_{\star}<1$, as the roles of the $x$ and $z$ axes are now reversed for the prolate figure. Formulas for other values of $\gamma_{\star}$, or stellar density fall-off, are given in Appendix A. We plot the logarithm of the virial ratio in Fig. 5 along with the ratio calculated from the M2M models. The line for $\gamma_{\star}=3$ agrees well with the M2M data. The green dashed line shows the virial ratio computed using the virial method. For a stellar density stratified on the same concentric ellipsoids as the darkmatter density, the virial ratio is simply a function of the shape of the ellipsoids and is independent of the radial density profile so the plotted line has a very simple functional form $[52,58,59]$.

It can be shown that along the sequence of models the total luminosity-averaged square velocity dispersion $\sigma_{\text {tot }}$ is constant. Therefore, for a given model the kinematic factor is the ratio of the total velocity dispersion to the line-ofsight velocity dispersion. When viewed down the $x$ axis, the kinematic correction factor is

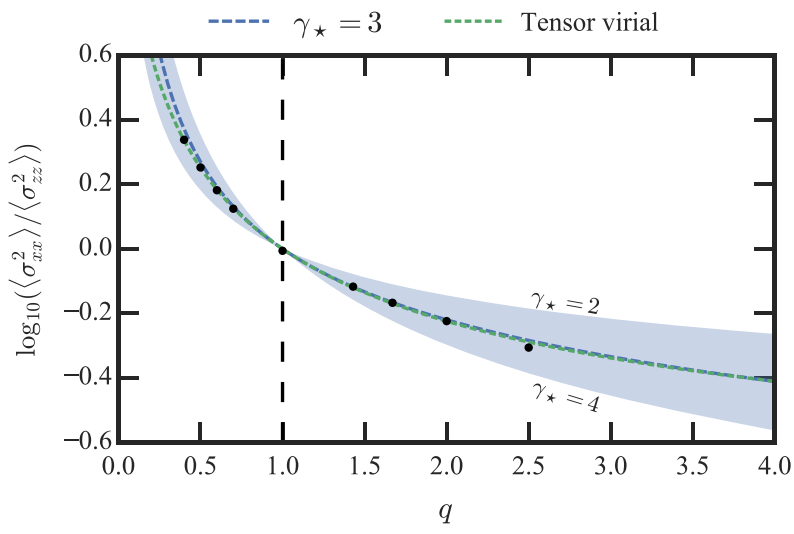

FIG. 5. Kinematic ratio for oblate and prolate figures. Each line shows the prediction from a stellar axisymmetric cusp with density slope $\gamma_{\star}$ flattened with axis ratio $q$ embedded in a halo also with flattening $q$. The black points show the numerical results from the M2M models. 


$$
J_{\text {kin,edge }}=\left(\frac{\left\langle\sigma_{\text {tot }}^{2}\right\rangle}{\left\langle\sigma_{\text {los }}^{2}\right\rangle}\right)^{2}=\left(\frac{2}{3}+\frac{\left\langle\sigma_{z z}^{2}\right\rangle}{3\left\langle\sigma_{x x}^{2}\right\rangle}\right)^{2} .
$$

This is the ratio of the squared velocity dispersion along the line of sight to the average value. This is smaller (larger) than unity for oblate (prolate) models. When viewed down the $z$ axis, the kinematic correction factor is

$$
J_{\text {kin,face }}=\left(\frac{1}{3}+\frac{2\left\langle\sigma_{x x}^{2}\right\rangle}{3\left\langle\sigma_{z z}^{2}\right\rangle}\right)^{2}
$$

This is smaller (larger) than unity for prolate (oblate) models. Note that, as the D-factors are proportional to $\sigma_{\text {tot }}^{2}$, the D-factor kinematic factor $D_{\text {kin }}=\sqrt{J_{\text {kin }}}$.

\section{Flat rotation curve models}

A simple but widely used model of a dark halo has the potential-density pair $[52,56]$

$$
\begin{aligned}
\rho_{\mathrm{DM}}(R, z) & =\frac{v_{0}^{2}}{4 \pi G q_{\phi}^{2}} \frac{\left(2 q_{\phi}^{2}+1\right) R_{\mathrm{d}}^{2}+R^{2}+z^{2}\left(2-q_{\phi}^{-2}\right)}{\left(R_{\mathrm{d}}{ }^{2}+R^{2}+z^{2} q_{\phi}^{2}\right)^{2}}, \\
\Phi_{\mathrm{DM}}(R, z) & =\frac{v_{0}^{2}}{2} \ln \left(R_{\mathrm{d}}^{2}+R^{2}+z^{2} q_{\phi}^{-2}\right) .
\end{aligned}
$$

Here, $v_{0}$ is a velocity scale that is the asymptotic value of the flat rotation curve, while $R_{\mathrm{d}}$ is the dark matter length scale and $q_{\phi}$ is the axis ratio of the equipotentials. The dark matter density is everywhere positive provided $q_{\phi}>1 / \sqrt{2}$, so the model can be oblate, spherical or prolate. Unless $q_{\phi}=1$, the flattening of the dark matter density changes with radius such that the oblate models become more oblate in the outskirts while the prolate models become more prolate. At large radii $q_{\phi}$ is related to the isodensity flattening $q$ via Eq. (20). The dark halo is cusped if $R_{\mathrm{d}}=0$, but the cusp is isothermal and so much more severe than in the NFW model.

The J-factor for the model viewed along the $z$ axis or symmetry axis is

$$
\begin{aligned}
\mathrm{J}= & \frac{v_{0}^{4}}{96 R_{\mathrm{d}} D^{2} G^{2} q_{\phi}^{3}}\left[3(1-y)-4 q_{\phi}^{2}\left(y^{3}-1\right)\right. \\
& \left.+q_{\phi}^{4}\left(8-3 y-2 y^{3}-3 y^{5}\right)\right],
\end{aligned}
$$

with $y=R_{d} / \sqrt{R_{d}^{2}+D^{2} \theta^{2}}$. At large angles, $y \rightarrow 0$ and so the asymptotic value is

$$
\mathrm{J} \rightarrow \frac{v_{0}^{4}}{96 R_{\mathrm{d}} D^{2} G^{2} q_{\phi}^{3}}\left[3+4 q_{\phi}^{2}+8 q_{\phi}^{4}\right] .
$$

Similarly, the D-factor for a model viewed along the $z$ axis is given by

$$
\mathrm{D}=\frac{v_{0}^{2} R_{d}}{G D^{2}} \frac{q_{\phi}\left(D \theta / R_{d}\right)^{2}}{\sqrt{1+\left(D \theta / R_{d}\right)^{2}}}
$$

Note that the total mass of the model is not finite so the $\mathrm{D}$-factor does not tend to a finite value as $\theta \rightarrow \infty$. Viewed edge on, two of the integrations for the J-factor are analytic, leaving a final integral over the spherical aperture to be performed numerically

$$
J(\theta \rightarrow \infty)=\frac{v_{0}^{4}}{1536 \pi R_{\mathrm{d}} D^{2} G^{2} q_{\phi}^{8}} \int_{0}^{2 \pi} d \phi \frac{G_{1}\left(q_{\phi}\right)+G_{2}\left(q_{\phi}\right) \cos (2 \phi)+G_{3}\left(q_{\phi}\right) \cos (4 \phi)}{\left(\cos ^{2} \phi+q_{\phi}^{-2} \sin ^{2} \phi\right)^{3}},
$$

$$
\begin{aligned}
& G_{1}\left(q_{\phi}\right)=120+280 \mathcal{Q}^{2}+221 \mathcal{Q}^{4}+64 \mathcal{Q}^{6}+9 \mathcal{Q}^{8}, \\
& G_{2}\left(q_{\phi}\right)=4 \mathcal{Q}^{2}\left(14+3 \mathcal{Q}^{2}\left(3+\mathcal{Q}^{2}\right)^{2}\right), \\
& G_{3}\left(q_{\phi}\right)=\mathcal{Q}^{4}\left(7+8 \mathcal{Q}^{2}+3 \mathcal{Q}^{4}\right),
\end{aligned}
$$

and $\mathcal{Q}^{2}=q_{\phi}^{2}-1$. The D-factor can also be expressed as a single quadrature but the expression is too bulky to present here. Into this dark halo, we embed a population of stars to model the $\mathrm{dSph}$, namely

$$
\rho(R, z)=\frac{\rho_{0} R_{\mathrm{c}}^{\beta_{\star}}}{\left(R_{\mathrm{c}}{ }^{2}+R^{2}+z^{2} q_{\star}^{-2}\right)^{\beta_{\star} / 2}} .
$$

Here, $\rho_{0}$ is a normalization constant, while $q$ is the axis ratio of the spheroidal isodensity contours. If $\beta_{\star}=5$, this is the familiar Plummer model. If the scale length of the stars $R_{\mathrm{c}}$ is equal to the scale length of the dark matter $R_{\mathrm{d}}$, and the flattening of the stellar density $q_{\star}$ is equal to the flattening of the dark matter equipotentials $q_{\phi}$, then the phase space distribution function is an isothermal [56]. We derive more general formulas below, but note that this simple limit enables an easy check of the correctness of our results.

As both the density and the potential are simple, we can calculate the velocity dispersions seen on viewing the stellar distribution along the short or long axis. We give the results for $\beta_{\star}=5$ here, and delegate other formulas to Appendix B. We begin by defining 


$$
\begin{aligned}
& \Delta_{1}^{2}=q_{\phi}^{2}-q_{\star}^{2}, \quad \Delta_{2}^{2}=R_{\mathrm{d}}{ }^{2}-R_{\mathrm{c}}{ }^{2}, \\
& \mathcal{D}^{2}=q_{\phi}^{2} R_{\mathrm{d}}^{2}-q_{\star}^{2} R_{\mathrm{c}}^{2} .
\end{aligned}
$$

Then the velocity dispersions are

$$
\begin{aligned}
\left\langle\sigma_{R R}^{2}\right\rangle= & \frac{v_{0}^{2} q_{\phi} R_{\mathrm{c}}^{2}}{\Delta_{1}^{4} \Delta_{2}^{4} \mathcal{D}^{2}}\left[2 \Delta_{1}^{4} R_{\mathrm{d}}{ }^{3} \mathcal{D} \operatorname{Arccosh}\left(\frac{q_{\phi} R_{\mathrm{d}}}{q_{\star} R_{\mathrm{c}}}\right)\right. \\
& -\Delta_{1} \mathcal{D}^{2}\left(2 q_{\phi}^{2} R_{\mathrm{d}}{ }^{2}+q_{\star}^{2}\left(R_{\mathrm{c}}{ }^{2}-3 R_{\mathrm{d}}^{2}\right)\right) \operatorname{Arccosh}\left(\frac{q_{\phi}}{q_{\star}}\right) \\
& \left.-q_{\phi} \Delta_{1}^{2} \Delta_{2}^{2} \mathcal{D}^{2}\right], \\
\left\langle\sigma_{z z}^{2}\right\rangle= & \frac{v_{0}^{2} q_{\star}^{2} R_{\mathrm{c}}^{2}}{\Delta_{1}^{4} \Delta_{2}^{2} \mathcal{D}^{2}}\left[q_{\star}^{2} \Delta_{1}^{2} \Delta_{2}^{2} \mathcal{D}^{2}-q_{\phi} \Delta_{1} \mathcal{D}^{4} \operatorname{Arccosh}\left(\frac{q_{\phi}}{q_{\star}}\right)\right. \\
& \left.+q_{\phi} \Delta_{1}^{4} \mathcal{D} R_{\mathrm{d}}{ }^{3} \operatorname{Arccosh}\left(\frac{q_{\phi} R_{\mathrm{d}}}{q_{\star} R_{\mathrm{c}}}\right)\right] .
\end{aligned}
$$

The formulas hold generally on using the identity (for $S<1)$

$$
\operatorname{Arccosh} S \equiv-i \arccos S .
$$

These formulas give the line-of-sight velocity dispersion of an axisymmetric Plummer model viewed along the short and long axes in a dark halo of arbitrary flattening and length scale. If $R_{\mathrm{c}}=R_{\mathrm{d}}$, then

$$
\begin{aligned}
\left\langle\sigma_{R R}^{2}\right\rangle= & \frac{v_{0}^{2} q_{\phi}}{4\left(q_{\phi}^{2}-q_{\star}^{2}\right)^{3}}\left[5 q_{\star}^{4} q_{\phi}-7 q_{\star}^{2} q_{\phi}^{3}+2 q_{\phi}^{5}\right. \\
& \left.+3 q_{\star}^{4}\left(q_{\phi}^{2}-q_{\star}^{2}\right)^{1 / 2} \operatorname{Arccosh}\left(\frac{q_{\phi}}{q}\right)\right], \\
\left\langle\sigma_{z z}^{2}\right\rangle= & \frac{v_{0}^{2} q_{\star}^{2}}{2\left(q_{\phi}^{2}-q_{\star}^{2}\right)^{3}}\left[q_{\phi}^{4}+q_{\phi}^{2} q_{\star}^{2}-2 q_{\star}^{4}\right. \\
& \left.-3 q_{\star}^{2} q_{\phi}\left(q_{\phi}^{2}-q_{\star}^{2}\right)^{1 / 2} \operatorname{Arccosh}\left(\frac{q_{\phi}}{q}\right)\right] .
\end{aligned}
$$

If additionally $q_{\star}=q_{\phi}$, then

$$
\left\langle\sigma_{R R}^{2}\right\rangle=\frac{2 v_{0}^{2}}{5}, \quad\left\langle\sigma_{z z}^{2}\right\rangle=\frac{v_{0}^{2}}{5} .
$$

With the line-of-sight velocity dispersion in hand, we can simply rescale the model so that the J-factors are computed for models with the same observables (line-of-sight velocity dispersion and half-light radius) as the flattenings and the ratio of dark to luminous scale length $R_{\mathrm{c}} / R_{\mathrm{d}}$ varies.

\section{Comparisons}

The models in Secs. IV B and IV C are complementary. The infinite cusps allow us to vary the central slope of the dark matter. The cored models allow us to vary the ratio of the luminous to the dark matter length scale. Taken together, a gamut of possibilities of dark halo cusps, density profiles and length scales can be swept out.

The base-10 logarithms of the correction factors (6) are plotted as a function of dark halo flattening $q$ in Fig. 6. The flattening of the stellar population $q_{\star}$ is the same as that of the dark halo $q$. For all plots we use the observed parameters of Reticulum II with a beam angle of $0.5^{\circ}$.

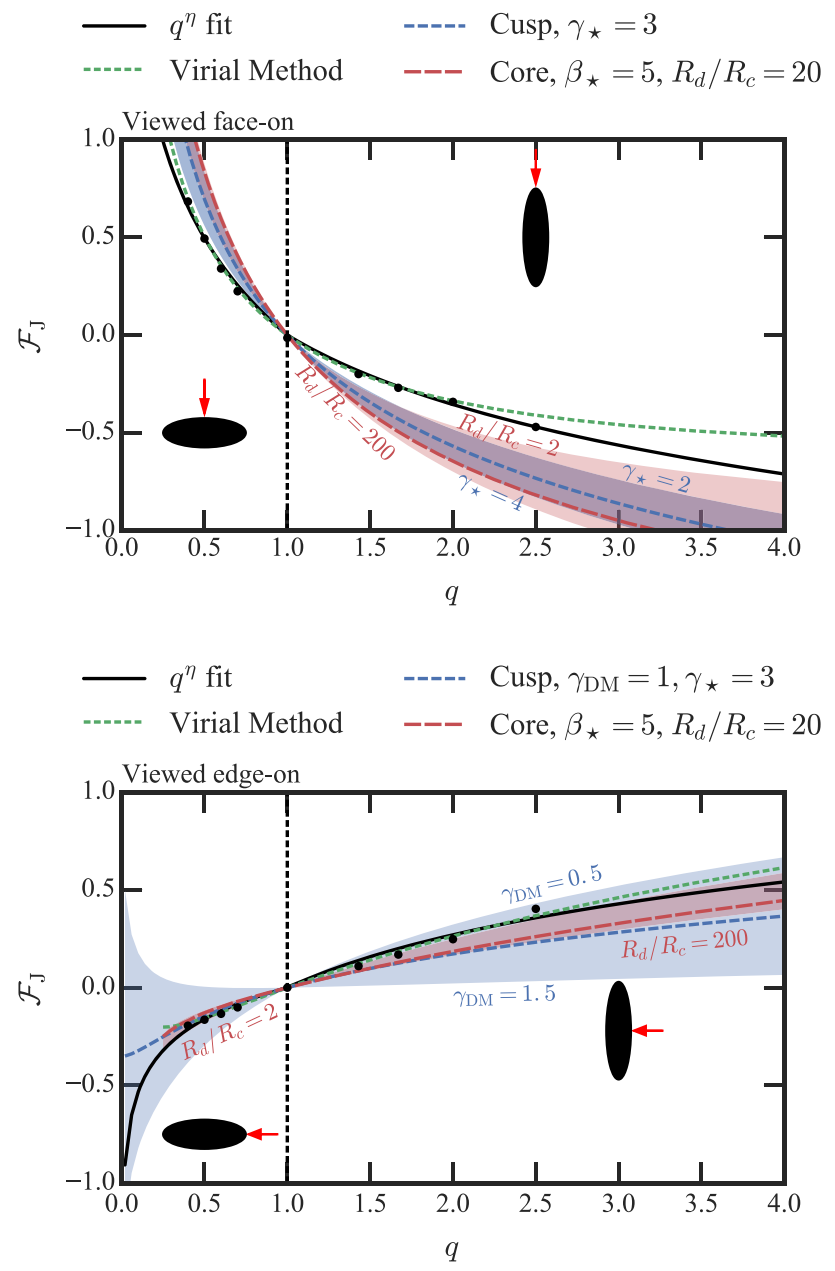

FIG. 6. J-factor correction factors for oblate and prolate figures viewed face on (top) and edge on (bottom). The black points show the numerical results from the M2M models of stellar Plummer models flattened with axis ratio $q$ embedded in NFW dark-matter halos also of axis ratio $q$. The dashed green line shows the results of the virial method of Sec. IV A. The blue band shows a range of axisymmetric cusp models from Sec. IV B. The central line corresponds to a model with $\gamma_{\mathrm{DM}}=1, \gamma_{\star}=3$. In the top panel, we have varied the slope of the light profile (note the face-on correction factor is independent of $\gamma_{\mathrm{DM}}$ in this case). In the bottom panel, we have varied the slope of the dark matter. In both panels, the red band shows a series of cored flat rotation curve models from Sec. IV C. The central line has an outer stellar density profile of $\beta_{\star}=5$ and a ratio of dark-matter to stellar scale radii of $R_{\mathrm{d}} / R_{\mathrm{c}}=20$. The band corresponds to varying this scale radii ratio by a factor of 10 . 
The correction factors are a function of the beam angle but we have found that this dependence is very weak and that the correction factors are essentially independent of the size of the dSph with respect to the beam size. Therefore, the reported correction factors are appropriate for all beam angles.

We show the correction factors for models viewed face on (top panels) and edge on (bottom panels). Any correction factor between these extremes should be possible as the inclination angle can be varied between these extremes. The correction factors computed from the M2M Plummer models in NFW halos with axis ratio $q$ are shown as black points. They are in good agreement with the results of the virial method of Sec. IV A applied to the self-same model, which are shown as a green curve. Note that the exception to this is the $q=2.5$ prolate model. As noted in Sec. II, the made-to-measure method in this case produces an equilibrium figure that significantly deviates from a spheroidally stratified Plummer model. We also show as blue bands the range of results for the axisymmetric cusps of Sec. IV B, in which both the slopes of the dark matter and stellar cusps are allowed to vary. Finally, the red band shows a series of cored flat rotation curve models from Sec. IV C. The central line has an outer stellar density slope of $\beta_{\star}=5$ and a ratio of dark-matter to stellar scale radii of $R_{\mathrm{d}} / R_{\mathrm{c}}=20$. The band corresponds to varying this scale radii ratio by a factor of 10 . As we move along the curves, the models have the same line-of-sight velocity dispersion and the same half-light radius. As the models make different assumptions as to the dark matter density and potential, we do not expect these curves to match up exactly with the M2M models, but it is encouraging that they all show similar trends.

When an oblate model is viewed along the short axis or face on, it appears circular, but there is always a boost to the J-factor. For flattenings of $q=q_{\star}=0.5$, this can be a factor of 3 boost over the spherical J-factor. When the model is viewed edge on, or along the long axis, then it appears flattened with isophotes of ellipticity $1-q_{\star}$. However, the geometric and kinematic corrections work in different directions, i.e., to boost and reduce the J-factor, respectively. The net effect is less significant than in the face-on case and is $\sim 0.1-0.2$ dex for $0.4<q<0.7$.

When a prolate model is viewed along the long or $z$ axis, it appears round. Here, the geometric and the kinematic corrections both diminish the J-factor. Although the model looks round on the sky, its J-factor can be substantially less than that computed by a spherical analysis. For example, if the true flattening is $q=q_{\star}=2.5$, then the J-factor is decreased by a factor $\lesssim 0.3$. When the model is viewed edge on, the isophotes have ellipticity $1-q_{\star}^{-1}$, and the kinematic and geometric factors act in opposite directions with the net result being a small boost. If the flattening is $q=q_{\star}=2.5$ the $\mathrm{J}$-factor is increased by $\sim 0.3$ dex over the spherical estimate.
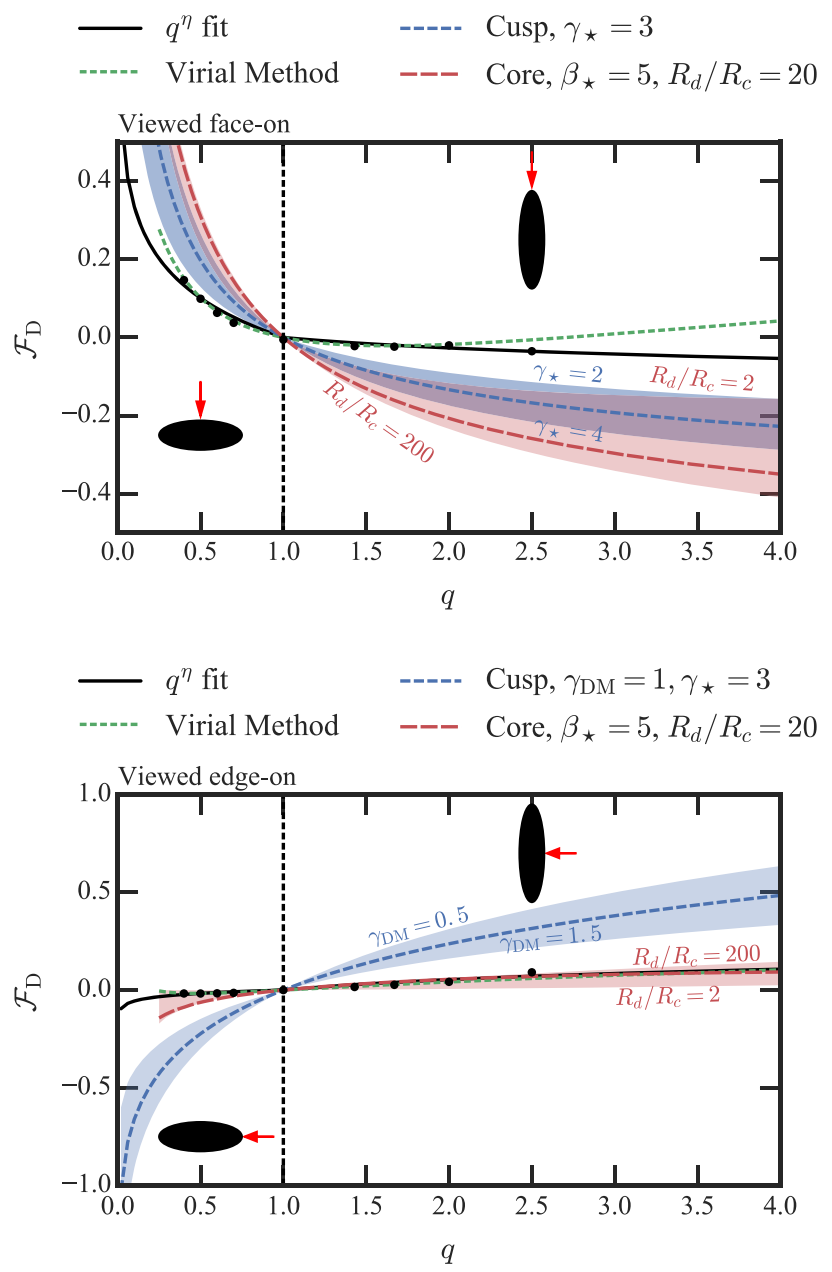

FIG. 7. D-factor correction factors for oblate and prolate figures viewed face on (top) and edge on (bottom). See the caption of Fig. 6 for details on each line.

The blue bands give an indication of how the correction factors vary as the dark-matter density slope is adjusted. We find steeper cusps give smaller corrections for models viewed edge on, but have no effect on the corrections for models viewed face on as the geometric factor is independent of $\gamma_{\mathrm{DM}}$. For the face-on case we see that making the slope of the stellar density profile steeper produces larger corrections to the J-factor.

From the red bands we observe that making the dark matter halo more extended (increasing $R_{\mathrm{d}} / R_{\mathrm{c}}$ ) produces larger corrections for the face-on case but smaller corrections for the edge-on case. The width of the red bands when the length scales $R_{\mathrm{c}} / R_{\mathrm{d}}$ are varied is at most 0.5 dex, even at the most extreme flattenings. Most of the Milky Way dSphs are rounder than $q_{\star}=0.5$. In this regime, the red band is thinner, and gives rise to an uncertainty of at most $\sim 0.25$ dex. This suggests that varying the concentration of the dark matter halo will not have a significant effect on the flattened J-factors. This is corroborated by experiments with the Plummer profile embedded in the NFW profile. 
Additionally, making the stellar density in these models fall off more rapidly increases the magnitude of the J-factor correction factors when viewing face on but decreases the magnitude of the correction when viewing edge on.

The equivalent results for the D-factors are shown in Fig. 7. The correction factors for the M2M models are all $\lesssim 0.2$ dex, and suggest that for most applications, the spherical approximation suffices for the D-factors. Note that in the face-on case, the cored models give a similar approximation of the correction factors as the cuspy models while for the edge-on case the cored models more faithfully represent the true correction factors than the cuspy models. Increasing the outer stellar density slope for the flat rotation curve models increases the magnitude of D-factor correction factors when viewing face on but has little effect for the edge-on case.

Finally, we note that for a $(\alpha, \beta, \gamma)$ stellar model of Eq. (3) embedded in another $(\alpha, \beta, \gamma)$ dark-matter model the $\mathrm{J}$-factor correction factors are very insensitive to the choice of the density slopes of the stellar and dark-matter distributions and the ratio of the stellar to dark-matter scale lengths. The same is broadly true for the D-factor correction factors except that the edge-on correction factor has a weak dependence with the outer slope of the dark matter profile. This is slightly at odds with the flat rotation curve model results but this may be due to the flat rotation curve models having a density flattening that varies with radius while the $(\alpha, \beta, \gamma)$ models have a constant density flattening.

\section{THE EFFECTS OF TRIAXIALITY}

Generically, we might expect the light and dark-matter distributions in dwarf spheroidals to be triaxial [60]. Triaxiality can introduce additional flattening (stretching) along the line of sight and so naturally increases (decreases) the J-factor and gives rise to larger (smaller) correction factors. Here we extend the formulas given in the previous section to account for intrinsic triaxial shapes. We begin by focusing on the infinite cusp models where some analytic progress can be made before moving on to consider more general density profiles.

We extend the models of Eq. (11) and introduce an intermediate-to-major axis ratio $p$ in addition to the minor-to-major axis ratio $q$. Here we restrict $q<p<1$ such that a prolate model has $p=q \neq 1$. It is conventional to use a triaxiality parameter $T$ to describe the figures defined by

$$
T=\frac{1-p^{2}}{1-q^{2}} .
$$

Note that figures with $T=0$ are oblate while those with $T=1$ are prolate. The density for the triaxial cusp models is
$\rho_{\mathrm{DM}}(m)=\frac{M_{\mathrm{h}}}{4 \pi p q m_{\mathrm{h}}^{3-\gamma_{\mathrm{DM}}}} \frac{3-\gamma_{\mathrm{DM}}}{m^{\gamma_{\mathrm{DM}}}}$ for $\quad m \leq r_{t}$,

and zero otherwise. $m^{2}=x^{2}+y^{2} p^{-2}+z^{2} q^{-2}$ and $r_{t}$ is a truncation ellipsoidal radius. When an infinite $\left(r_{t} \rightarrow \infty\right)$ model is viewed along the $z$ axis, the observed flattening is $p$ and the geometric factor is a combination of Eqs. (15) and (16) such that

$\mathrm{J}_{\mathrm{geo}, \mathrm{Z}}=\frac{p^{2-\gamma_{\mathrm{DM}}}}{2 \pi p^{2} q} \int_{0}^{2 \pi} d \theta\left(\cos ^{2} \theta+p^{-2} \sin ^{2} \theta\right)^{1 / 2-\gamma_{\mathrm{DM}}}$.

In this case, the observed major-axis length corresponds to the intrinsic model scale radius. for $\gamma_{\mathrm{DM}} \leq 2$, the integral is a monotonic function of $q$ that is greater than unity for $q<1$ and less than unity for $q>1$. If viewed along the $y$ axis the observed flattening is $q$ and the J-factor is given by

$\mathrm{J}_{\mathrm{geo}, \mathrm{y}}=\frac{q^{2-\gamma_{\mathrm{DM}}}}{2 \pi q^{2} p} \int_{0}^{2 \pi} d \theta\left(\cos ^{2} \theta+q^{-2} \sin ^{2} \theta\right)^{1 / 2-\gamma_{\mathrm{DM}}}$,

and again the observed major-axis length coincides with the intrinsic model scale radius. When viewed along the major axis, the observed flattening is $q / p$ and the observed majoraxis length coincides with the intermediate axis so the resultant measured scale length must be scaled by a factor of $1 / p$. This gives rise to a geometric factor of

$\mathrm{J}_{\mathrm{geo}, \mathrm{X}}=\frac{(q / p)^{2-\gamma_{\mathrm{DM}}}}{2 \pi(q / p)^{2} p} \int_{0}^{2 \pi} d \theta\left(\cos ^{2} \theta+(q / p)^{-2} \sin ^{2} \theta\right)^{1 / 2-\gamma_{\mathrm{DM}}}$.

As with the axisymmetric case, the infinite cusps have limited use and it is more practical to use models with finite truncation ellipsoidal radii $r_{t}<D \theta$. In this case, the geometric factors are given by

$$
\begin{aligned}
\mathrm{J}_{\mathrm{geo}, \mathrm{x}} & =(q p)^{1-\gamma_{\mathrm{DM}}}, \\
\mathrm{J}_{\mathrm{geo}, \mathrm{y}} & =q^{1-\gamma_{\mathrm{DM}}} / p, \\
\mathrm{~J}_{\mathrm{geo}, \mathrm{z}} & =p^{1-\gamma_{\mathrm{DM}}} / q .
\end{aligned}
$$

For the models that produce a finite J-factor $\left(\gamma_{\mathrm{DM}}<3 / 2\right)$, we find $\mathrm{J}_{\mathrm{geo}, \mathrm{x}}<\mathrm{J}_{\mathrm{geo}, \mathrm{y}}<\mathrm{J}_{\mathrm{geo}, \mathrm{z}}$. For the astrophysically motivated case of $\gamma_{\mathrm{DM}}=1$ the geometric factors are simply $\mathrm{J}_{\mathrm{geo}, \mathrm{x}}=1, \mathrm{~J}_{\mathrm{geo}, \mathrm{y}}=1 / p$ and $\mathrm{J}_{\mathrm{geo}, \mathrm{z}}=1 / q$.

If the infinite model is observed along a line of sight oriented with spherical polar angles $(\varphi, \vartheta)$ with respect to the intrinsic Cartesian coordinates of the model, the geometric factor must be computed with the full threedimensional integrals as

$$
\mathrm{J}_{\text {geo }}=\frac{1}{\mathrm{~J}_{\text {sph }} D^{2}} \int_{-\infty}^{\infty} \mathrm{d} z^{\prime} \int_{0}^{2 \pi} \mathrm{d} \theta \int_{0}^{D \alpha} \mathrm{d} R R \rho_{\mathrm{DM}^{2}}(\boldsymbol{x}),
$$


where $\boldsymbol{x}=R \cos \theta \hat{\boldsymbol{\varphi}}+R \sin \theta \hat{\boldsymbol{\vartheta}}+z^{\prime} \hat{\boldsymbol{r}}$ with $(\hat{\boldsymbol{r}}, \hat{\boldsymbol{\varphi}}, \hat{\boldsymbol{\vartheta}})$ being the set of spherical polar unit vectors. Making the model finite with $r_{t}<D \theta$ produces $\mathrm{J} / \mathrm{J}_{\mathrm{sph}}=1 /(p q)$. However, for this general viewing angle the calculation of the observed scale radius seems intractable. The kinematic factors $\mathbf{J}_{\text {kin }}=\left(\left\langle\sigma_{\text {tot }}^{2}\right\rangle /\left\langle\sigma_{\text {los }}^{2}\right\rangle\right)^{2}$ can be derived for these more general viewing angles as

$\mathrm{J}_{\text {kin }}=\left[\frac{1}{3} \frac{1+f_{1}+f_{2}}{\cos ^{2} \vartheta+f_{1} \sin ^{2} \vartheta \cos ^{2} \varphi+f_{2} \sin ^{2} \vartheta \sin ^{2} \varphi}\right]^{2}$,

where

$f_{1}=\frac{\left\langle\sigma_{x x}^{2}\right\rangle}{\left\langle\sigma_{z z}^{2}\right\rangle}=\frac{\int_{0}^{\pi} \mathrm{d} \theta \int_{0}^{2 \pi} \mathrm{d} \phi F(\theta, \phi) \sin ^{3} \theta \cos ^{2} \phi}{\int_{0}^{\pi} \mathrm{d} \theta \int_{0}^{2 \pi} \mathrm{d} \phi F(\theta, \phi) \sin \theta \cos ^{2} \theta}>f_{2}$

$f_{2}=\frac{\left\langle\sigma_{y y}^{2}\right\rangle}{\left\langle\sigma_{z z}^{2}\right\rangle}=\frac{\int_{0}^{\pi} \mathrm{d} \theta \int_{0}^{2 \pi} \mathrm{d} \phi F(\theta, \phi) \sin ^{3} \theta \sin ^{2} \phi}{\int_{0}^{\pi} \mathrm{d} \theta \int_{0}^{2 \pi} \mathrm{d} \phi F(\theta, \phi) \sin \theta \cos ^{2} \theta}>1$,

and

$F(\theta, \phi)=\left(\sin ^{2} \theta \cos ^{2} \phi+P_{\star}^{2} \sin ^{2} \theta \sin ^{2} \phi+Q_{\star}^{2} \cos ^{2} \theta\right)^{-\gamma_{\mathrm{DM}} / 2}$.

Here $P_{\star}=p_{\phi} / p$ and $Q_{\star}=q_{\phi} / q$ with $p_{\phi}$ and $q_{\phi}$ being the axis ratios of the dark matter potential. We see that when viewing down the major axis $(\vartheta=\pi / 2, \varphi=0)$ the kinematic correction factor is less than unity while viewing down the minor axis $(\vartheta=0, \varphi=0)$ produces a kinematic correction factor greater than unity. Generally, we find that $\mathrm{J}_{\text {kin, } \mathrm{x}}<\mathrm{J}_{\text {kin,y }}<\mathrm{J}_{\text {kin, } \mathrm{z}}$ such that the total correction factors for $\gamma_{\mathrm{DM}}<3 / 2$ obey the hierarchy $\mathcal{F}_{\mathrm{J}, \mathrm{x}}<\mathcal{F}_{\mathrm{J}, \mathrm{y}}<\mathcal{F}_{\mathrm{J}, \mathrm{Z}}$. We have found that the effects of triaxiality seem to be in accordance with our expectation from the axisymmetric case. When there is additional flattening along the line of sight the geometric and kinematic correction factors combine to increase the correction factor, while additional stretching decreases the correction factor.

We now compute general triaxial correction factors using the method of Sec. IVA. We show an example of the J-factor correction factors for the Reticulum II model presented in Sec. II but with stellar minor-to-major axis ratio $q=0.4$ and stellar intermediate-to-major axis ratio $p=0.73$. We assume the dark-matter distribution is flattened in the same way as the stellar distribution. This model has triaxiality parameter $T=0.55$, (which was deemed the best fit to the Local Group dSphs by Ref. [60]). The base-10 logarithm of the correction factor for all viewing angles is given in Fig. 8. We see that, in agreement with the simple predictions from the infinite cusp models, the largest correction factor occurs when the model is viewed down the short axis (the $z$ axis) while the smallest is when viewing down the long axis (the $x$ axis). The black contours on the sphere show lines of constant
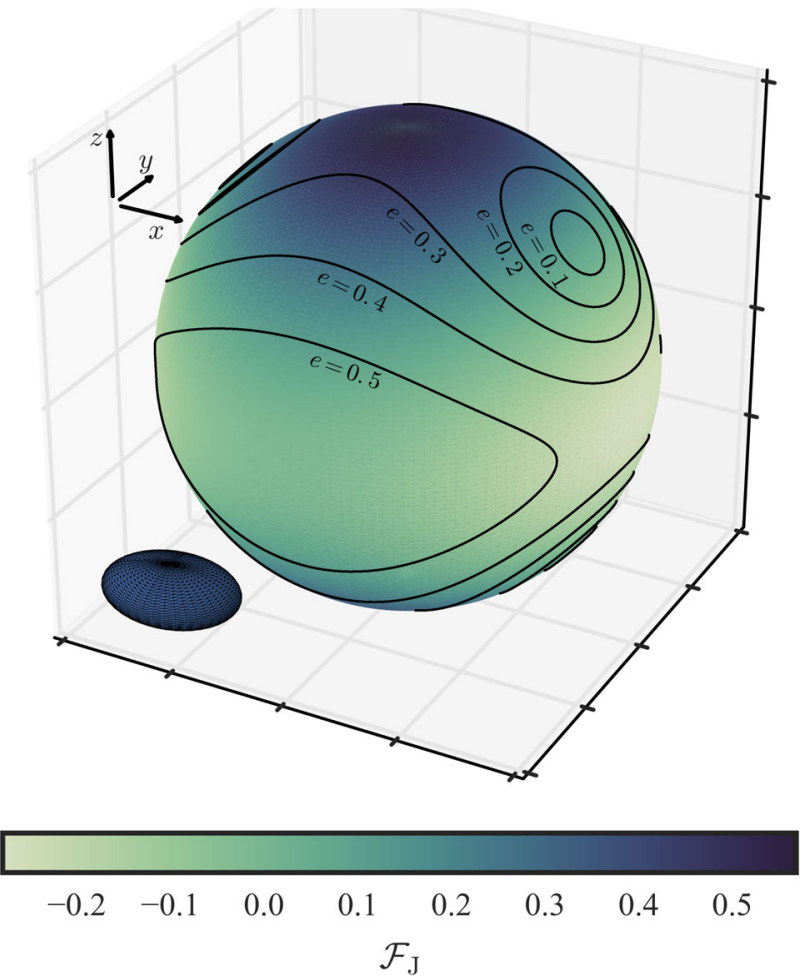

FIG. 8. J-factor correction factors for a triaxial model of Reticulum II: each point on the sphere is colored by the correction factor when viewing the model along the radial vector that passes through that point. The black contours show the observed ellipticity when viewed from that direction. The small ellipsoid shows an isodensity contour for the considered model which has axis ratios $p=0.73$ and $q=0.4$ in both the stellar and darkmatter distributions. The largest correction factor is achieved when viewing the model down the short axis $(z)$ while the smallest correction factor is achieved when viewing the model down the long axis $(x)$. When viewing down the intermediate axis (y) the observed ellipticity matches that of Reticulum II.

observed ellipticity. We see that for this figure an observed ellipticity of 0.3 gives rise to a variation in the correction factor of 0.6 dex.

In conclusion, additional flattening along the line of sight can lead to an increase in the correction factors. For a general triaxial figure the largest correction factor is obtained when viewing the model down the short axis while the smallest correction factor is yielded when viewing the model down the long axis.

\section{INTRINSIC SHAPES AND AXIS ALIGNMENTS OF DWARF SPHEROIDAL GALAXIES}

We have presented corrections to the J- and D-factors based on the assumption that the dSphs are prolate or oblate figures with axes aligned with the line of sight. Such configurations are quite unlikely as we anticipate that generically the $\mathrm{dSph}$ principal axes are misaligned with the line of sight. In this section we will discuss what is 
known regarding the intrinsic shapes of the $\mathrm{dSphs}$ and how this translates into observed properties via their axes alignment with respect to the line of sight.

For a given individual galaxy, we have a couple of probes of its intrinsic shape [61-63]. The first of these is the presence of isophotal twisting, that is the change in the orientation of the major axis of the isodensity contours with on-sky distance from the galaxy center. Isophotal twisting is a clear signature of a triaxial figure with varying axis ratios with radius, although isophotal twisting may also be caused by tidal effects [64]. Another indicator of triaxiality is evidence of kinematic misalignment between the axis of rotation and the minor axis of the projected density.

For entire populations of galaxies, progress can be made by analyzing statistics of the population (e.g., Ref. [49]). Recently, Ref. [60] demonstrated that under the assumption that the intrinsic axes of the dSphs are randomly oriented, the Local Group dSph population is best reproduced by triaxial models with mean triaxiality $\bar{T}=0.55_{-0.22}^{+0.21}$ and a mean intrinsic ellipticity $[E=1-(c / a)]$ of $\bar{E}=0.51_{-0.06}^{+0.07}$. The assumption of random orientation is perhaps to be questioned, particularly for the Milky Way dSphs. Darkmatter only simulations $[65,66]$ have demonstrated that the major axes of subhalos tend to be aligned with the radial direction to the center of their host halo and this picture has been corroborated when baryons have been included [67]. The main exception to this is near the subhalo's pericentric passage where the major axis is briefly aligned perpendicular to the radial direction.

Most of the dSphs are distant enough for the radial direction and our line of sight to approximately coincide, which suggests that for many dSphs the observed flattening corresponds to the intermediate-to-minor axis ratio and that there is significant stretching of the dSphs along the line of sight. As demonstrated in this paper, this gives rise to overestimates of the J-factors from spherical analyses for the prolate face-on models and for the triaxial model viewed down the major axis.

Based on this discussion, we now compute the expected $\mathrm{J}$ correction factors with their associated uncertainties under a number of assumptions regarding the intrinsic shape and alignment of the dSphs. We use the EMCEE package from Ref. [68] to draw 500 samples of $(T, E, \vartheta, \varphi)$ i.e. the triaxiality, the intrinsic ellipticity and the two viewing angles. Our likelihood is the distribution of the observed ellipticity for each dSph given by, for instance, Eqs. (A1) and (A2) of Ref. [49]. For those dSphs with upper bounds on their ellipticity we use a normal distribution with mean zero and standard deviation of half the upper bound. We consider three different prior distributions on the parameters $(T, E, \vartheta, \varphi)$ :

(1) Uniform (U): $\quad T \sim \mathcal{U}(0,1), \quad E \sim \mathcal{U}(0,0.95)$, $\cos \vartheta \sim \mathcal{U}(0,1), \varphi \sim \mathcal{U}(0, \pi / 2)$.

(2) Viewing down the major axis (R): $T \sim \mathcal{U}(0,1)$, $E \sim \mathcal{U}(0,0.95), \vartheta \sim \mathcal{N}(\pi / 2,0.1 \mathrm{rad}), \varphi \sim \mathcal{N}(0,0.1 \mathrm{rad})$.
(3) Sanchez-Janssen et al. [60] priors (T): $T \sim$ $\mathcal{N}(0.55,0.04), \quad E \sim \mathcal{N}(0.51,0.12), \quad \cos \vartheta \sim \mathcal{U}(0,1)$, $\varphi \sim \mathcal{U}(0, \pi / 2)$,

where $\mathcal{U}(a, b)$ is a uniform distribution from $a$ to $b$ and $\mathcal{N}(\mu, \sigma)$ is a normal distribution with mean $\mu$ and standard deviation $\sigma$. For each sample we compute the base-10 logarithm of the correction factor $\mathcal{F}_{\mathrm{J}}$ to construct a distribution of correction factors.

In Fig. 9, we show the full one-dimensional distributions of the correction factors for Reticulum II. All three prior assumptions produce a correction factor distribution that peaks near zero. The broadest distribution corresponds to the case where uniform priors have been adopted in all parameters. In this case, the largest correction factors correspond to models with high intrinsic ellipticity $E$ viewed down the short axis. These models have triaxiality $T$ close to unity so they are near prolate models. The smallest correction factors correspond to models with low intrinsic ellipticity viewed down the long axis.

For the prior assumption that we are viewing along the major axis, we find the median correction factor peaks at $\sim-0.2$ dex. For this prior assumption, there is an approximate one-to-one relationship between $T$ and $E$ as well as $T$ and $\mathcal{F}_{\mathrm{J}}$. Models with smaller $T$ correspond to smaller $E$ and hence smaller amplitude correction factors as these models are approximately edge-on oblate, while larger $T$ and larger $E$ produce larger amplitude negative correction factors as these models are nearer prolate stretched along the line of sight. For the prior assumption that the models have some fixed triaxiality and intrinsic ellipticity, the largest correction factors correspond to viewing angles nearer the short axis and the smallest correction factors correspond to viewing angles closer to the long axis.

The medians and $\pm 1 \sigma$ error bars of the correction factors for all the dSphs computed for the three prior assumptions are given in Table V. This information is also displayed in Fig. 10. If we assume the $\mathrm{dSphs}$ are preferentially viewed

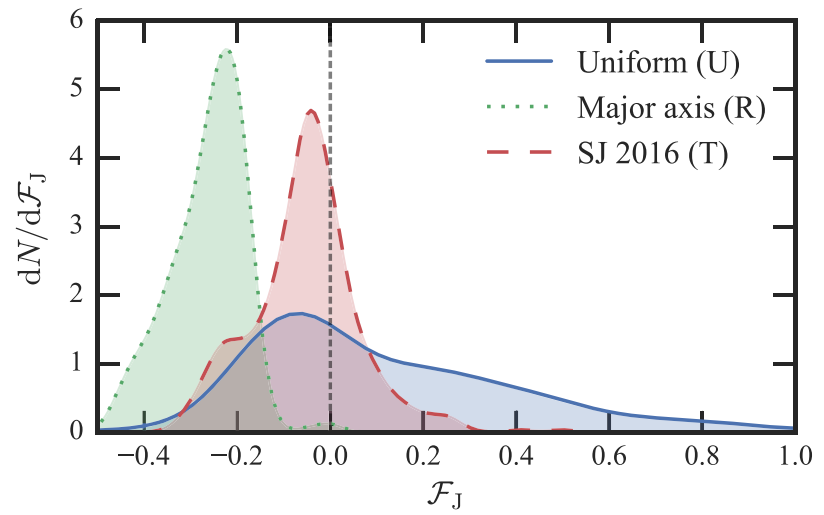

FIG. 9. Distribution of the base-10 logarithm of the J-factor correction factors for triaxial models of Reticulum II under three different assumptions on the prior distributions of the intrinsic triaxiality, ellipticity and viewing angles as described in the text. 
TABLE V. Median and $\pm 1 \sigma \mathrm{J}$ correction factors for the known dSphs for three different assumptions about the intrinsic shapes and alignments. The first correction factor (marked "U") uses flat uniform priors on the triaxiality, minor-to-major axis ratio and viewing angles. The second (marked " $\mathrm{R}$ ") uses a normal prior on the viewing angles centered on $(\theta=\pi / 2, \phi=0)$ with width $5^{\circ}$. The third (marked "T") uses a normal prior on the triaxiality and minor-to-major axis ratios with means $(0.55,0.49)$ and widths $(0.04,0.12)$ (based on the fits to the Local Group dSphs of Sanchez-Janssen et al. [60]).

\begin{tabular}{|c|c|c|c|c|}
\hline Name & Ellipticity & $\mathcal{F}_{\mathrm{J}, U}$ & $\mathcal{F}_{\mathrm{J}, R}$ & $\mathcal{F}_{\mathrm{J}, T}$ \\
\hline Hydra II & $0.01_{-0.01}^{+0.20}$ & $-0.01_{-0.02}^{+0.10}$ & $-0.05_{-0.12}^{+0.04}$ & $0.07_{-0.17}^{+0.19}$ \\
\hline Leo $\mathrm{T}$ & $<0.10$ & $-0.00_{-0.02}^{+0.07}$ & $-0.06_{-0.13}^{+0.04}$ & $0.03_{-0.06}^{+0.08}$ \\
\hline Leo II & $0.13_{-0.05}^{+0.05}$ & $0.02_{-0.07}^{+0.18}$ & $-0.11_{-0.14}^{+0.06}$ & $0.09_{-0.15}^{+0.15}$ \\
\hline Segue 2 & $0.15_{-0.10}^{+0.10}$ & $0.01_{-0.07}^{+0.25}$ & $-0.11_{-0.13}^{+0.07}$ & $0.09_{-0.17}^{+0.21}$ \\
\hline Leo I & $0.21_{-0.03}^{+0.03}$ & $0.03_{-0.11}^{+0.28}$ & $-0.16_{-0.15}^{+0.07}$ & $0.13_{-0.23}^{+0.20}$ \\
\hline Horologium I & $<0.28$ & $0.00_{-0.04}^{+0.13}$ & $-0.08_{-0.13}^{+0.06}$ & $0.09_{-0.15}^{+0.19}$ \\
\hline Fornax & $0.30_{-0.01}^{+0.01}$ & $0.04_{-0.13}^{+0.38}$ & $-0.19_{-0.15}^{+0.07}$ & $0.06_{-0.21}^{+0.33}$ \\
\hline Draco & $0.31_{-0.02}^{+0.02}$ & $0.04_{-0.13}^{+0.28}$ & $-0.19_{-0.13}^{+0.06}$ & $0.05_{-0.17}^{+0.30}$ \\
\hline Sculptor & $0.32_{-0.03}^{+0.03}$ & $0.04_{-0.15}^{+0.36}$ & $-0.21_{-0.13}^{+0.08}$ & $0.04_{-0.19}^{+0.28}$ \\
\hline Carina & $0.33_{-0.05}^{+0.05}$ & $0.06_{-0.15}^{+0.31}$ & $-0.20_{-0.13}^{+0.08}$ & $0.03_{-0.18}^{+0.27}$ \\
\hline Sextans & $0.35_{-0.05}^{+0.05}$ & $0.06_{-0.16}^{+0.27}$ & $-0.21_{-0.12}^{+0.08}$ & $0.00_{-0.16}^{+0.24}$ \\
\hline Coma Berenices & $0.38_{-0.14}^{+0.14}$ & $0.04_{-0.15}^{+0.32}$ & $-0.21_{-0.13}^{+0.08}$ & $0.03_{-0.16}^{+0.28}$ \\
\hline Boötes I & $0.39_{-0.06}^{+0.06}$ & $0.04_{-0.15}^{+0.42}$ & $-0.22_{-0.12}^{+0.07}$ & $0.00_{-0.18}^{+0.24}$ \\
\hline Canes Venatici I & $0.39_{-0.03}^{+0.03}$ & $0.07_{-0.17}^{+0.27}$ & $-0.22_{-0.13}^{+0.07}$ & $-0.01_{-0.14}^{+0.17}$ \\
\hline Tucana II & $0.39_{-0.20}^{+0.10}$ & $0.03_{-0.13}^{+0.33}$ & $-0.20_{-0.14}^{+0.09}$ & $0.01_{-0.14}^{+0.22}$ \\
\hline Pisces II & $0.40_{-0.10}^{+0.10}$ & $0.07_{-0.18}^{+0.36}$ & $-0.20_{-0.12}^{+0.07}$ & $0.00_{-0.16}^{+0.21}$ \\
\hline Grus I & $0.41_{-0.28}^{+0.20}$ & $0.01_{-0.12}^{+0.29}$ & $-0.21_{-0.12}^{+0.11}$ & $0.01_{-0.14}^{+0.24}$ \\
\hline Willman 1 & $0.47_{-0.08}^{+0.08}$ & $0.08_{-0.21}^{+0.33}$ & $-0.24_{-0.12}^{+0.07}$ & $-0.01_{-0.13}^{+0.16}$ \\
\hline Segue 1 & $0.48_{-0.13}^{+0.13}$ & $0.05_{-0.18}^{+0.35}$ & $-0.23_{-0.12}^{+0.06}$ & $-0.01_{-0.16}^{+0.19}$ \\
\hline Leo IV & $0.49_{-0.11}^{+0.11}$ & $0.07_{-0.20}^{+0.32}$ & $-0.24_{-0.11}^{+0.07}$ & $-0.02_{-0.15}^{+0.15}$ \\
\hline Leo V & $0.50_{-0.15}^{+0.15}$ & $0.05_{-0.18}^{+0.33}$ & $-0.24_{-0.11}^{+0.08}$ & $-0.01_{-0.15}^{+0.20}$ \\
\hline Canes Venatici II & $0.52_{-0.11}^{+0.11}$ & $0.09_{-0.22}^{+0.33}$ & $-0.23_{-0.11}^{+0.06}$ & $-0.02_{-0.13}^{+0.15}$ \\
\hline Ursa Minor & $0.56_{-0.05}^{+0.05}$ & $0.03_{-0.18}^{+0.41}$ & $-0.25_{-0.12}^{+0.06}$ & $-0.03_{-0.11}^{+0.08}$ \\
\hline Reticulum II & $0.59_{-0.03}^{+0.02}$ & $0.08_{-0.23}^{+0.29}$ & $-0.26_{-0.10}^{+0.07}$ & $-0.04_{-0.12}^{+0.08}$ \\
\hline Ursa Major II & $0.63_{-0.05}^{+0.05}$ & $0.06_{-0.22}^{+0.32}$ & $-0.26_{-0.09}^{+0.06}$ & $-0.04_{-0.13}^{+0.07}$ \\
\hline Hercules & $0.68_{-0.08}^{+0.08}$ & $0.08_{-0.23}^{+0.35}$ & $-0.24_{-0.09}^{+0.06}$ & $-0.05_{-0.10}^{+0.07}$ \\
\hline Ursa Major I & $0.80_{-0.04}^{+0.04}$ & $0.02_{-0.19}^{+0.39}$ & $-0.22_{-0.08}^{+0.08}$ & $-0.06_{-0.10}^{+0.06}$ \\
\hline
\end{tabular}

along the major axis, the median correction factor is less than unity and is weakly decreasing with increasing ellipticity. As the dSphs become more flattened on the sky, they are forced to become more extended along the line of sight and so the J-factor decreases. The medians of the correction factors for dSphs with small ellipticity under the uniform prior assumption is around 0.05 dex and the upper error bars are in general larger than the lower error bars but the spread encompasses zero. The median shift is due to the asymmetry in the correction factors between oblate and prolate models seen in Fig. 6. This suggests that all J-factors are underestimated by $\sim 10$ percent but naturally this conclusion is very sensitive to the exact prior assumptions. For small ellipticity, the spreads in the correction factors for the uniform and fixed shape priors are approximately equal with the spread weakly increasing with increasing ellipticity for the uniform case. For the fixed shape prior the spread decreases at large ellipticity as models viewed down the minor axis become inconsistent with the observed ellipticity. We have fitted a simple relation to the uncertainty in the correction factors from the uniform priors $\Delta \mathcal{F}_{\mathrm{J}}$ (the average of the $\pm 1 \sigma$ uncertainties) as a function of ellipticity as

$$
\Delta \mathcal{F}_{\mathrm{J}} \approx 0.4 \sqrt{\epsilon}
$$




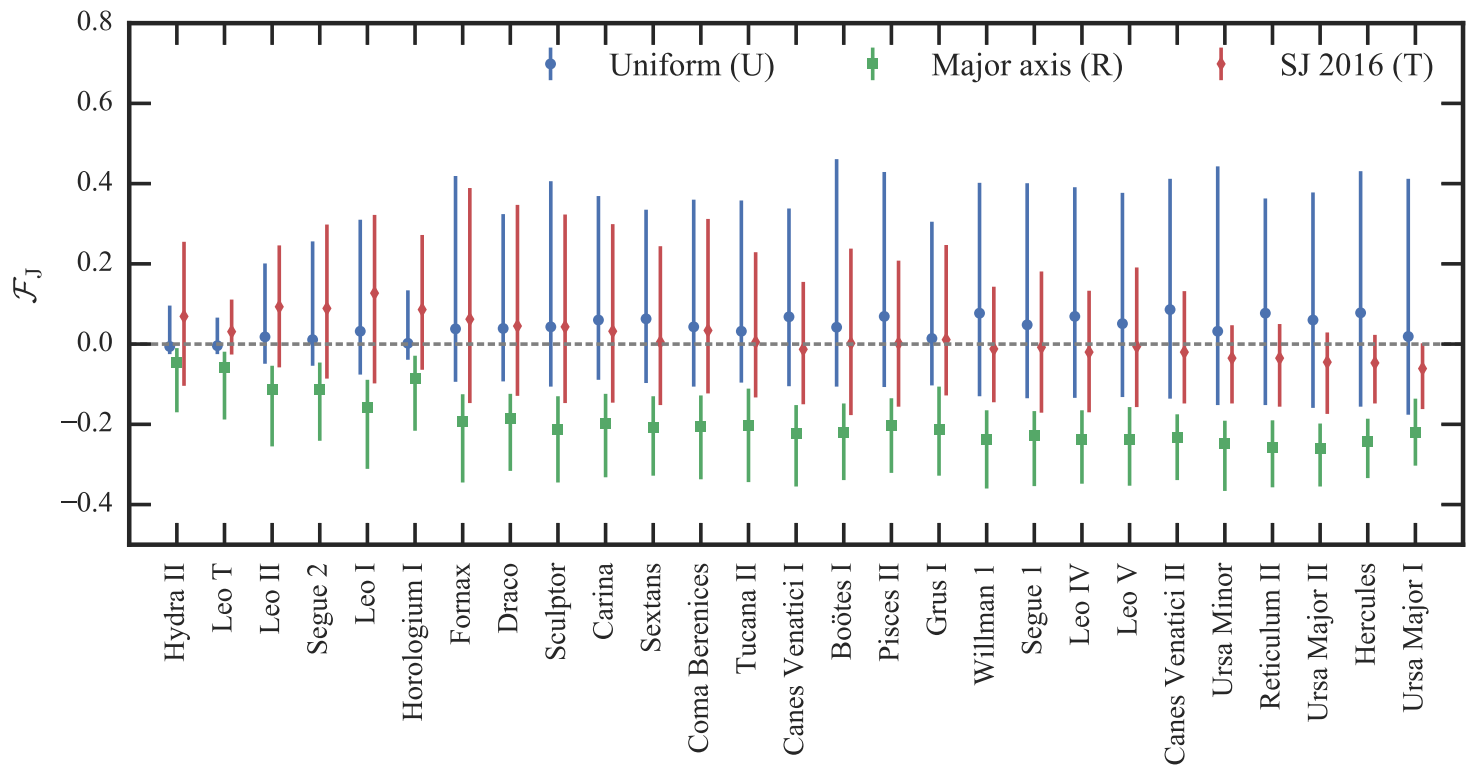

FIG. 10. Medians and $\pm 1 \sigma$ error bars for the base-10 logarithms of the J-factor correction factors for all the dSphs ranked by their ellipticity. The three different error bars correspond to three different prior assumptions regarding the intrinsic shapes and alignments of the dSphs as described in the text.

which gives a fractional uncertainty in the J-factor of

$$
\frac{\Delta \mathrm{J}}{\mathrm{J}} \approx 10^{0.4 \sqrt{\epsilon}}-1
$$

This expression gives 50 percent uncertainty for $\epsilon \approx 0.2$, a factor of 1.8 uncertainty for $\epsilon \approx 0.4$ and a factor of 2.3 uncertainty for $\epsilon \approx 0.6$. For small $\epsilon, \Delta \mathrm{J} / \mathrm{J} \approx 0.9 \epsilon$. We conclude for a typical dSph ellipticity of 0.4 there is approximately a factor of 2 uncertainty in the J-factors due to the unknown triaxiality and alignment of the dSph.

\section{DISCUSSION AND CONCLUSIONS}

Flattening is a crucial attribute of a dwarf spheroidal galaxy. Both the dark halo and the stellar distribution can be flattened. The ultrafaint $\mathrm{dSphs}$ have many fewer baryons than the classical dwarfs so it is anticipated that feedback effects have a weaker effect on the shape of the dark matter distribution in the ultrafaints. Therefore, for the ultrafaints, a flattened stellar distribution probably corresponds to a flattened dark matter distribution. Of these ultrafaints, Reticulum II is an interesting object as it is particularly nearby and also one of the most highly flattened of all the ultrafaints, at least as judged by the stellar light. On these grounds, we might well expect that flattening may provide an explanation as to why a gamma-ray signal may have been seen towards Reticulum II as opposed to other ultrafaints.

We have explored the impact of flattening on the $\mathrm{J}$ - and D-factors, which control the expected dark matter annihilation and decay signals from the dSphs. The effects of flattening on these factors can be decomposed into two separate corrections: the geometric and the kinematic factors. The first of these corresponds to the increase (decrease) in dark-matter density produced by squeezing (stretching) the models. The latter corresponds to how the observed velocity dispersion relates to the total velocity dispersion or the enclosed dark matter mass. When viewing oblate (prolate) models face on, these two factors act together to increase (decrease) the J-factor over a spherical analysis, whereas, when viewing these models edge on, the two factors compete and result in a decrease (increase) in the J-factor over a spherical analysis.

We have used made-to-measure techniques $[37,38]$ to build numerical equilibrium models of Reticulum II. These reproduce the flattened shape, the major-axis length and the line-of-sight velocity dispersion of Reticulum II. For the models with a prolate dark matter halo with ellipticity $\sim 0.6$ viewed edge on, flattening could cause an additional amplification of 2-2.5 for Reticulum II over that expected for spherical dark halos. This factor could be still larger if the stellar profile falls off more slowly than a Plummer law (which could increase the kinematic factor). It could also be larger if the dark halo of Reticulum II is triaxial (as anticipated from dark-matter-only simulations) and hence more flattened along the line of sight. However, this scenario is disfavored by dark-matter simulations with and without baryons that produce subhalos which preferentially point towards the center of their host halo and so we might anticipate $\mathrm{dSphs}$ to be elongated along the line of sight.

We corroborated the results of the made-to-measure simulations with a simpler virial method that allows for more rapid calculation of the correction factors for general 
geometries. A simple fitting relation has been provided for rapid estimation of the correction factors for the oblate and prolate cases. Additionally, we have inspected two cases where some analytic progress can be made in the computation of the J-factors. This has allowed us to characterize how the correction factors change as a function of the stellar and dark-matter distributions. We found that the correction factors for the made-to-measure models agree well with the trends seen in the analytic models.

We used our models to estimate the J-factors for the $\mathrm{dSphs}$ under the assumption that the figures are aligned with the line of sight and are either oblate or prolate. The ranking of the J-factors of the $\mathrm{dSphs}$ is slightly altered when accounting for flattening under the assumption that all the $\mathrm{dSphs}$ are either prolate or oblate. Typical correction factors for a dSph with ellipticity 0.4 are 0.75 in the oblate case and 1.6 in the prolate case. We also demonstrated that the corrections to the D-factors are much smaller than the scatter in the spherical D-factor from the other observables. For instance, a dSph with ellipticity 0.4 has a D-factor correction factor of 0.97 in the oblate case and 1.1 in the prolate case. Therefore, we concluded that flattening is unimportant for D-factor computation.

We concluded our discussion of the effects of flattening by computing correction factors for triaxial figures. The findings from the axisymmetric cases were found to simply extend when considering triaxiality. The largest J-factor correction factor corresponds to viewing the figure along the minor axis, while the smallest corresponds to viewing the figure along the major axis. We found that for a Reticulum II-like model the J-factor correction factor varies by a factor of $\sim 6-10$ as one changes the viewing angle. For a fixed observed ellipticity, the correction factor can vary by a factor of $\sim 4$. We demonstrated that for the known dSphs the uncertainty in the correction factors due to unknown triaxiality increases with the observed ellipticity of the $\mathrm{dSph}$ and is typically a factor of 2 for $\epsilon \sim 0.4$. If all $\mathrm{dSphs}$ have their major axes aligned with the line of sight (as suggested by some simulations), the correction factors decrease as a function of observed ellipticity and are typically a factor of $1 / 2$ for $0.4 \lesssim \epsilon \lesssim 0.6$.

Deviations from sphericity in both the light profile and the dark matter are important. This suggests fundamental limitations to the spherical Jeans modeling which is common in the field (although see Ref. [31] for J-factors computed using axisymmetric Jeans modeling). In particular, increasingly sophisticated statistical techniques $[69,70]$ will fail to include an inherent uncertainty if the assumption of a spherical stellar density profile in a spherical dark halo breaks down. The uncertainties, which are different for different dSphs, must be accounted for in joint analyses of multiple dSphs.

Spherical Jeans modeling is probably most useful for large classical dwarf spheroidal galaxies that look nearly round (such as Leo I or Fornax). It ignores important uncertainties for the ultrafaints, which is unfortunate as these are the most promising targets of all for indirect dark matter detection. We hope that the work presented here-a systematic foray into the domain of flattening-is the beginning of a systematic exploration of more general flattened and triaxial dark halo shapes.

Finally, this study was partly inspired by the gamma-ray detection [17] toward the very flattened ultrafaint, Reticulum II. Our work demonstrates that Reticulum II could have a J-factor that is higher than spherical analyses suggest if it is a prolate figure. However, the correction for the prolate shape does not make Reticulum II stand out as the $\mathrm{dSph}$ with the highest J-factor nor does a lack of signal from the other $\mathrm{dSphs}$ create any tension, irrespective of the shapes of the other dSphs. If, however, Reticulum II is an oblate figure the J-factor is lower than that found through spherical analyses and a lack of signal from the other dSphs may give rise to some tension if the other dSphs (such as Ursa Minor) are prolate. More generically we have demonstrated that unknown triaxiality produces an uncertainty in the J-factor for Reticulum II of a factor of $\sim 2$.

In general, we have shown that the effect of flattening on expected dark matter annihilation fluxes cannot be ignored. Indeed, flattening can shift expected signals by amounts larger than error bars due to current velocity dispersion measurements. These currently unknown shifts change the ranking of $\mathrm{dSph}$ targets for gamma-ray experiments. However, if the orientations of the Milky Way dSphs can be determined, the results presented here can help pin down relative J-factors and allow tests of dark matter explanations of gamma-ray detections.

\section{ACKNOWLEDGMENTS}

J. L. S. acknowledges financial support from the Science and Technology Facilities Council (STFC) of the United Kingdom. Figure 1 was produced using the PYNBODY package [71].

\section{APPENDIX A: VIRIAL RATIOS FOR CUSPED MODELS}

In Sec. IV B we presented the virial ratio $\left\langle\sigma_{x x}^{2}\right\rangle /\left\langle\sigma_{z z}^{2}\right\rangle$ for a flattened cusped model with density slope $\gamma_{\star}=3$. Here we provide formulas for other values of $\gamma_{\star}$. For $\gamma_{\star}=2$, the virial ratio is

$$
\frac{\left\langle\sigma_{x x}^{2}\right\rangle}{\left\langle\sigma_{z z}^{2}\right\rangle}=\frac{Q_{\star} T(\mathcal{Q})-\mathcal{Q}}{2[\mathcal{Q}-T(\mathcal{Q})]},
$$

where $T=$ Arctanh for $Q_{\star}<1$ and $T=$ Arctan for $Q_{\star}>1$, and $\mathcal{Q}$ is defined in Eq. (22). For $\gamma_{\star}=4$, the virial ratio is

$$
\frac{\left\langle\sigma_{x x}^{2}\right\rangle}{\left\langle\sigma_{z z}^{2}\right\rangle}=\frac{Q_{\star}\left[\left(\mathcal{Q}^{2}-1\right) T(\mathcal{Q})+\mathcal{Q}\right]}{2\left[Q_{\star} T(\mathcal{Q})-\mathcal{Q}\right]}
$$


where we have corrected a typographical error in Eq. (21) of Ref. [54]. For $\gamma_{\star}=5$, the virial ratio is simply

$$
\frac{\left\langle\sigma_{x x}^{2}\right\rangle}{\left\langle\sigma_{z z}^{2}\right\rangle}=Q_{\star} .
$$

A plot of the ratios as a function of flattening are given in Fig. 1 in Ref. [54]

\section{APPENDIX B: VELOCITY DISPERSIONS FOR FLAT ROTATION CURVE MODELS}

In Sec. IV C, we presented formulas for the velocity dispersions of a cored stellar profile with outer density slope $\beta_{\star}=5$ embedded in a cored dark matter density profile. Here we provide equivalent formulas for the cases $\beta_{\star}=4$ and $\beta_{\star}=6$.

Let us recall the definitions

$$
\Delta_{1}^{2}=q_{\phi}^{2}-q_{\star}^{2}, \quad \Delta_{2}^{2}=R_{\mathrm{d}}^{2}-R_{\mathrm{c}}^{2},
$$

and let us introduce the function

$F=\frac{1}{\Delta_{1} \Delta_{2}}\left[\operatorname{Arctan}\left(\frac{R_{\mathrm{d}} \Delta_{1}}{q_{\star} \Delta_{2}}\right)-\operatorname{Arctan}\left(\frac{R_{\mathrm{c}} \Delta_{1}}{q_{\phi} \Delta_{2}}\right)\right]$.

Notice that if $R_{\mathrm{c}}>R_{\mathrm{d}}$ or $q_{\star}>q$, this function remains well defined on using the identity (for $S<0$ )

$$
\frac{1}{\sqrt{S}} \operatorname{Arctan} \sqrt{S} \equiv \frac{1}{\sqrt{-S}} \operatorname{Arctanh} \sqrt{-S}
$$

For the case $\beta_{\star}=4$,

$$
\begin{aligned}
&\left\langle\sigma_{R R}^{2}\right\rangle= \frac{v_{0}^{2} q_{\phi} R_{\mathrm{c}}}{\Delta_{1}^{2} \Delta_{2}^{2}}\left[\left(q_{\phi}^{2} R_{\mathrm{d}}^{2}+q_{\star}^{2}\left(R_{\mathrm{c}}^{2}-2 R_{\mathrm{d}}^{2}\right)\right) F\right. \\
&\left.-q_{\phi} R_{\mathrm{c}}+q_{\star} R_{\mathrm{d}}\right] \\
&\left\langle\sigma_{z z}^{2}\right\rangle=\frac{v_{0}^{2} q_{\star}^{2} R_{\mathrm{c}}}{\Delta_{1}^{2}}\left[q_{\phi} F-\frac{q_{\star}}{q_{\star} R_{\mathrm{c}}+q_{\phi} R_{\mathrm{d}}}\right] .
\end{aligned}
$$

If $R_{\mathrm{c}}=R_{\mathrm{d}}$, then the velocity dispersions are a lot simpler. A careful Taylor expansion gives

$$
\begin{aligned}
\left\langle\sigma_{R R}^{2}\right\rangle & =\frac{2 q_{\phi}\left(2 q_{\star}+q_{\phi}\right) v_{0}^{2}}{3\left(q_{\star}+q_{\phi}\right)^{2}}, \\
\left\langle\sigma_{z z}^{2}\right\rangle & =\frac{q_{\star}^{2} v_{0}^{2}}{\left(q_{\star}+q_{\phi}\right)^{2}}
\end{aligned}
$$

so that when $q_{\star}=q_{\phi}$, we obtain

$$
\left\langle\sigma_{R R}^{2}\right\rangle=\frac{v_{0}^{2}}{2}, \quad\left\langle\sigma_{z z}^{2}\right\rangle=\frac{v_{0}^{2}}{4} .
$$

Finally, we give the results for $\beta_{\star}=6$,

$$
\begin{aligned}
\left\langle\sigma_{R R}^{2}\right\rangle= & \frac{v_{0}^{2} q_{\phi} R_{\mathrm{c}}^{2}}{\Delta_{1}^{2} \Delta_{2}^{4}}\left[\frac{R_{\mathrm{d}}\left(R_{\mathrm{c}}^{2}+2 R_{\mathrm{d}}^{2}\right) \Delta_{1}^{2}-q_{\star} q_{\phi} R_{\mathrm{c}} \Delta_{2}^{2}}{q_{\star} R_{\mathrm{c}}+q_{\phi} R_{\mathrm{d}}}\right. \\
& \left.-R_{\mathrm{c}}\left(3 q_{\phi}^{2} R_{\mathrm{d}}^{2}+q_{\star}^{2}\left(R_{\mathrm{c}}^{2}-4 R_{\mathrm{d}}^{2}\right)\right) F\right], \\
\left\langle\sigma_{z z}^{2}\right\rangle= & \frac{v_{0}^{2} q_{\star}^{2} R_{\mathrm{c}}^{2}}{\Delta_{1}^{2} \Delta_{2}^{2}}\left[-q_{\phi} R_{\mathrm{c}} F+\frac{q_{\star} q_{\phi} R_{\mathrm{c}} R_{\mathrm{d}}+q_{\phi}^{2} R_{\mathrm{d}}{ }^{2}-q_{\star}^{2} \Delta_{2}^{2}}{\left(q_{\star} R_{\mathrm{c}}+q_{\phi} R_{\mathrm{d}}\right)^{2}}\right] .
\end{aligned}
$$

If $R_{\mathrm{c}}=R_{\mathrm{d}}$, then

$$
\begin{aligned}
\left\langle\sigma_{R R}^{2}\right\rangle & =2 v_{0}^{2} \frac{q_{\phi}\left(8 q_{\star}^{2}+9 q_{\star} q_{\phi}+3 q_{\phi}^{2}\right)}{15\left(q+q_{\phi}\right)^{3}}, \\
\left\langle\sigma_{z z}^{2}\right\rangle & =v_{0}^{2} \frac{q_{\star}^{2}\left(3 q_{\star}+q_{\phi}\right)}{3\left(q_{\star}+q_{\phi}\right)^{3}}
\end{aligned}
$$

and if additionally $q_{\star}=q_{\phi}$, we recover

$$
\left\langle\sigma_{R R}^{2}\right\rangle=\frac{v_{0}^{2}}{3}, \quad\left\langle\sigma_{z z}^{2}\right\rangle=\frac{v_{0}^{2}}{6} .
$$

[1] A. Geringer-Sameth and S. M. Koushiappas, Phys. Rev. Lett. 107, 241303 (2011).

[2] M. Ackermann et al., Phys. Rev. Lett. 107, 241302 (2011).

[3] M. Ackermann et al., Phys. Rev. D 89, 042001 (2014).

[4] A. Geringer-Sameth, S. M. Koushiappas, and M. G. Walker, Phys. Rev. D 91, 083535 (2015).

[5] M. Ackermann et al., Phys. Rev. Lett. 115, 231301 (2015).
[6] E. Aliu et al., Phys. Rev. D 85, 062001 (2012).

[7] J. Aleksić et al., J. Cosmol. Astropart. Phys. 02 (2014) 008.

[8] A. Abramowski et al., Phys. Rev. D 90, 112012 (2014).

[9] MAGIC Collaboration, J. Cosmol. Astropart. Phys. 02 (2016) 039.

[10] B. Zitzer (for the VERITAS Collaboration), arXiv: 1509.01105. 
[11] J. Carr et al., arXiv:1508.06128.

[12] S. E. Koposov, V. Belokurov, G. Torrealba, and N. W. Evans, Astrophys. J. 805, 130 (2015).

[13] K. Bechtol et al., Astrophys. J. 807, 50 (2015).

[14] B. P. M. Laevens et al., Astrophys. J. Lett. 802, L18 (2015).

[15] A. Drlica-Wagner et al., Astrophys. J. 813, 109 (2015).

[16] C. He, K. Bechtol, A. P. Hearin, and D. Hooper, Phys. Rev. D 91, 063515 (2015).

[17] A. Geringer-Sameth, M. G. Walker, S. M. Koushiappas, S. E. Koposov, V. Belokurov, G. Torrealba, and N. W. Evans, Phys. Rev. Lett. 115, 081101 (2015).

[18] A. Drlica-Wagner et al., Astrophys. J. Lett. 809, L4 (2015).

[19] D. Hooper and T. Linden, J. Cosmol. Astropart. Phys. 09 (2015) 016.

[20] S. Li, Y.-F. Liang, K.-K. Duan, Z.-Q. Shen, X. Huang, X. Li, Y.-Z. Fan, N.-H. Liao, L. Feng, and J. Chang, Phys. Rev. D 93, 043518 (2016).

[21] A. Geringer-Sameth, S. M. Koushiappas, and M. Walker, Astrophys. J. 801, 74 (2015).

[22] V. Bonnivard et al., Mon. Not. R. Astron. Soc. 453, 849 (2015).

[23] A. Ibarra, D. Tran, and C. Weniger, Int. J. Mod. Phys. A 28, 1330040 (2013).

[24] N. W. Evans, J. L. Sanders, and A. Geringer-Sameth, Phys. Rev. D 93, 103512 (2016).

[25] M. G. Walker, M. Mateo, E. W. Olszewski, J. Peñarrubia, N. Wyn Evans, and G. Gilmore, Astrophys. J. 704, 1274 (2009).

[26] J. Wolf, G. D. Martinez, J. S. Bullock, M. Kaplinghat, M. Geha, R. R. Muñoz, J. D. Simon, and F. F. Avedo, Mon. Not. R. Astron. Soc. 406, 1220 (2010).

[27] A. J. Deason, V. Belokurov, N. W. Evans, L. L. Watkins, and M. Fellhauer, Mon. Not. R. Astron. Soc. 425, L101 (2012).

[28] N. F. Martin, J. T. A. de Jong, and H.-W. Rix, Astrophys. J. 684, 1075 (2008).

[29] D. B. Zucker et al., Astrophys. J. 650, L41 (2006).

[30] V. Bonnivard, C. Combet, D. Maurin, and M. G. Walker, Mon. Not. R. Astron. Soc. 446, 3002 (2015).

[31] K. Hayashi, K. Ichikawa, S. Matsumoto, M. Ibe, M. N. Ishigaki, and H. Sugai, arXiv:1603.08046.

[32] M. G. Abadi, J. F. Navarro, M. Fardal, A. Babul, and M. Steinmetz, Mon. Not. R. Astron. Soc. 407, 435 (2010).

[33] M. Zemp, O. Y. Gnedin, N. Y. Gnedin, and A. V. Kravtsov, Astrophys. J. 748, 54 (2012).

[34] Y. P. Jing and Y. Suto, Astrophys. J. 574, 538 (2002).

[35] B. Allgood, R. A. Flores, J. R. Primack, A. V. Kravtsov, R. H. Wechsler, A. Faltenbacher, and J. S. Bullock, Mon. Not. R. Astron. Soc. 367, 1781 (2006).

[36] A. Tenneti, R. Mandelbaum, T. Di Matteo, Y. Feng, and N. Khandai, Mon. Not. R. Astron. Soc. 441, 470 (2014).

[37] D. Syer and S. Tremaine, Mon. Not. R. Astron. Soc. 282, 223 (1996).

[38] W. Dehnen, Mon. Not. R. Astron. Soc. 395, 1079 (2009).

[39] M. Irwin and D. Hatzidimitriou, Mon. Not. R. Astron. Soc. 277, 1354 (1995).

[40] A. Agnello and N. W. Evans, Astrophys. J. Lett. 754, L39 (2012)
[41] N. C. Amorisco and N. W. Evans, Mon. Not. R. Astron. Soc. 411, 2118 (2011).

[42] S. E. Koposov et al., Astrophys. J. 811, 62 (2015).

[43] A. W. McConnachie, Astron. J. 144, 4 (2012).

[44] V. Belokurov et al., Astrophys. J.712, L103 (2010).

[45] N. F. Martin et al., Astrophys. J. 804, L5 (2015).

[46] B. Willman, M. Geha, J. Strader, L. E. Strigari, J. D. Simon, E. Kirby, N. Ho, and A. Warres, Astron. J. 142, 128 (2011).

[47] M. Niederste-Ostholt, V. Belokurov, N. W. Evans, G. Gilmore, R. F. G. Wyse, and J. E. Norris, Mon. Not. R. Astron. Soc. 398, 1771 (2009).

[48] V. Bonnivard, D. Maurin, and M. G. Walker, arXiv:1506.08209.

[49] A.-M. Weijmans et al., Mon. Not. R. Astron. Soc. 444, 3340 (2014).

[50] M. Cappellari, Mon. Not. R. Astron. Soc. 390, 71 (2008).

[51] N. W. Evans, J. An, A. Bowden, and A. A. Williams, Mon. Not. R. Astron. Soc. 450, 846 (2015).

[52] J. Binney and S. Tremaine, Galactic Dynamics, 2nd ed. (Princeton University, Princeton, NJ, 2008).

[53] J. Binney, Mon. Not. R. Astron. Soc. 183, 501 (1978).

[54] A. Agnello and N. W. Evans, Mon. Not. R. Astron. Soc. 422, 1767 (2012).

[55] S. Chandrasekhar, Ellipsoidal Figures of Equilibrium (Yale University, New Haven, CT, 1969).

[56] N. W. Evans, Mon. Not. R. Astron. Soc. 260, 191 (1993).

[57] A. Bowden, V. Belokurov, and N. W. Evans, Mon. Not. R. Astron. Soc. 449, 1391 (2015).

[58] P. H. Roberts, Astrophys. J. 136, 1108 (1962).

[59] S. D. M. White, Mon. Not. R. Astron. Soc. 237, 51P (1989).

[60] R. Sanchez-Janssen et al., arXiv:1602.00012.

[61] M. Franx, G. Illingworth, and T. de Zeeuw, Astrophys. J. 383, 112 (1991).

[62] T. S. Statler, Astrophys. J. 425, 500 (1994).

[63] R. C. E. van den Bosch and G. van de Ven, Mon. Not. R. Astron. Soc. 398, 1117 (2009).

[64] J. Kormendy, in Morphology and Dynamics of Galaxies; Proceedings of the Twelfth Advanced Course, Saas-Fee, Switzerland, March 29-April 3, 1982 (Sauverny, Switzerland, Observatoire de Geneve, 1983), p. 113.

[65] M. Kuhlen, J. Diemand, and P. Madau, Astrophys. J. 671, 1135 (2007).

[66] C. Barber, E. Starkenburg, J. F. Navarro, and A. W. McConnachie, Mon. Not. R. Astron. Soc. 447, 1112 (2015).

[67] A. Knebe, N. I. Libeskind, S. R. Knollmann, G. Yepes, S. Gottlöber, and Y. Hoffman, Mon. Not. R. Astron. Soc. 405, 1119 (2010).

[68] D. Foreman-Mackey, A. Conley, W. Meierjurgen Farr, D. W. Hogg, D. Long, P. Marshall, A. Price-Whelan, J. Sanders, and J. Zuntz, EMCEE: The MCMC Hammer, http:// ascl.net/1303.002.

[69] G. D. Martinez, Mon. Not. R. Astron. Soc. 451, 2524 (2015)

[70] J. Conrad, Astropart. Phys. 62, 165 (2015).

[71] A. Pontzen, R. Roškar, G. Stinson, and R. Woods, PYNBODY: N-Body/SPH analysis for python, http://ascl . net/1305.002. 